\title{
Excess diacylglycerol at the endoplasmic reticulum disrupts endomembrane homeostasis and autophagy
}

\author{
Dan Li ${ }^{1}$, Shu-Gao Yang ${ }^{2}$, Cheng-Wen He ${ }^{1}$, Zheng-Tan Zhang ${ }^{1}$, Yongheng Liang ${ }^{3}$, Hui Li $i^{1}$, Jing Zhu' ${ }^{1}$ Xiong Su², \\ Qingqiu Gong ${ }^{1 *}$ and Zhiping Xie ${ }^{1 *}$ (D)
}

\begin{abstract}
Background: When stressed, eukaryotic cells produce triacylglycerol (TAG) to store nutrients and mobilize autophagy to combat internal damage. We and others previously reported that in yeast, elimination of TAG synthesizing enzymes inhibits autophagy under nitrogen starvation, yet the underlying mechanism has remained elusive.

Results: Here, we show that disruption of TAG synthesis led to diacylglycerol (DAG) accumulation and its relocation from the vacuolar membrane to the endoplasmic reticulum (ER). We further show that, beyond autophagy, ERaccumulated DAG caused severe defects in the endomembrane system, including disturbing the balance of ER-Golgi protein trafficking, manifesting in bulging of ER and loss of the Golgi apparatus. Genetic or chemical manipulations that increase consumption or decrease supply of DAG reversed these defects. In contrast, increased amounts of precursors of glycerolipid synthesis, including phosphatidic acid and free fatty acids, did not replicate the effects of excess DAG. We also provide evidence that the observed endomembrane defects do not rely on Golgi-produced DAG, Pkc1 signaling, or the unfolded protein response.

Conclusions: This work identifies DAG as the critical lipid molecule responsible for autophagy inhibition under condition of defective TAG synthesis and demonstrates the disruption of ER and Golgi function by excess DAG as the potential cause of the autophagy defect.
\end{abstract}

Keywords: Phospholipid, Glycerolipid, Intracellular trafficking, Organelle, Autophagy

\section{Background}

Lipids are essential building blocks of life. Polar lipids, including phospholipids, sphingolipids, and sterols, are major constituents of cellular membranes. Neutral lipids, in particular TAG, are employed as a storage medium of carbon nutrients $[1,2]$. Storage lipid synthesis is induced

\footnotetext{
* Correspondence: xsu@suda.edu.cn; gongqingqiu@sjtu.edu.cn; zxie@sjtu.edu.cn

${ }^{1}$ State Key Laboratory of Microbial Metabolism \& Joint International Research Laboratory of Metabolic and Developmental Sciences, School of Life Sciences and Biotechnology, Shanghai Jiao Tong University, \#800 Dong-Chuan Road, Shanghai 200240, People's Republic of China

${ }^{2}$ School of Biology and Basic Medical Sciences, Soochow University, Suzhou, Jiangsu, People's Republic of China

Full list of author information is available at the end of the article
}

not only in the presence of nutrient surplus, but also in response to stress [3-6]. However, the amount of lipids that a cell or an organism can utilize and tolerate is limited. Too much lipid, from either environmental intake or de novo synthesis, can disrupt cellular homeostasis, leading to ER stress, ROS production, and cell death [7-9].

Recent studies revealed a close association of lipid metabolism with autophagy. Autophagy is a stress response pathway that utilizes double-membrane autophagosomes to clean up obsolete intracellular materials in eukaryotes $[10,11]$. Autophagosomes are formed from precursor membrane sacs, known as phagophores or isolation membranes. The phagophore is thought to emerge from

(c) The Author(s). 2020 Open Access This article is licensed under a Creative Commons Attribution 4.0 International License, which permits use, sharing, adaptation, distribution and reproduction in any medium or format, as long as you give appropriate credit to the original author(s) and the source, provide a link to the Creative Commons licence, and indicate if changes were made. The images or other third party material in this article are included in the article's Creative Commons licence, unless indicated otherwise in a credit line to the material. If material is not included in the article's Creative Commons licence and your intended use is not permitted by statutory regulation or exceeds the permitted use, you will need to obtain permission directly from the copyright holder. To view a copy of this licence, visit http://creativecommons.org/licenses/by/4.0/ The Creative Commons Public Domain Dedication waiver (http://creativecommons.org/publicdomain/zero/1.0/) applies to the data made available in this article, unless otherwise stated in a credit line to the data. 
a specialized region of ER [12-14]. The close contact between the phagophore and the ER facilitates lipid channeling by lipid transfer proteins $[15,16]$. Furthermore, anterograde trafficking out of the ER via COPII-coated vesicles is essential for autophagy [17-19]. This and many other vesicular trafficking pathways are reprogramed under stress conditions to connect the autophagy machinery with the rest of the endomembrane system, including the ER, Golgi, and endocytic compartments [20, 21]. Finally, the formation of autophagosomes appears to be coordinated with localized lipid biosynthesis, linking membrane dynamics with primary metabolism [22-24].

In yeast Saccharomyces cerevisiae, autophagy is strongly induced by nitrogen starvation [25]. Under the same condition, yeast cells continue glucose consumption and upregulate TAG production [26], which is analogous to observations in starved mammalian cells [27, 28]. The last step in TAG synthesis is catalyzed by two acyltransferases, Dga1 and Lro1 (Additional file 1: Fig. S1A) [29-31]. We and others have previously shown that elimination of both enzymes led to severe inhibition of autophagy [26, 32-34]. Different models have been proposed to explain this phenomenon, mainly from the perspective of fatty acid (FA) supply. In the present study, we set out to answer two key questions: first, what is the nature of the autophagy defect, and second, whether the disruption can be traced to a single causal factor while being consistent with established data.

\section{Results}

\section{Block of TAG synthesis disrupts the endomembrane system}

As the process of autophagy relies on membrane and protein input from the rest of the endomembrane system through direct contacts and vesicular trafficking pathways [20], we first analyzed the morphology of major membrane-based organelles in order to understand the na-

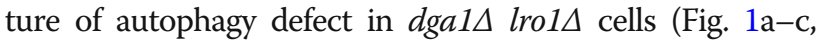
Additional file 1: Fig. S2). Markers of peroxisomes, late endosomes, and vacuoles displayed distribution patterns comparable to those in wild-type cells (Additional file 1: Fig. S2). In contrast, we noticed substantial alterations in the ER, Golgi, and mitochondria (Fig. 1a-c, Additional file 1: Fig. S2). As dga1 lro1 $\Delta$ cells entered nitrogen starvation, bulbous structures sprouted from the ER, the multi-puncta pattern of Golgi marker proteins vanished, and mitochondria became fragmented. The same changes were observed using multiple marker proteins of the same organelles (Fig. 1a, Additional file 1: Fig. S2), which suggest that an overall alteration in the conditions of the labeled organelles, rather than that of individual proteins, occurred in dga1 $\Delta$ lro1 $\Delta$ cells. The changes in organelle morphology happened progressively. The pace of these changes coincided with the occurrence of autophagy inhibition, as indicated by the reduction in the number of GFP-Atg8 positive autophagic structures (Fig. 1a, d). Colocalization analysis revealed that the bulbs contained both ER and Golgi proteins (Fig. 2a). They were not strongly stained by BODIPY (Fig. 2b), indicating that the bulbs are separate from lipid droplets, which also frequently associate with the ER. The size of most bulbs was visibly larger than that of regular autophagosomes. In live cell super resolution microscopy, we could easily see ring-like structure in wild-type cells expressing GFP-Atg8. Under the same condition, few ER bulbs in

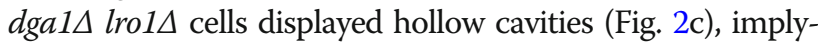
ing that the structures contain membrane proteins and potentially membrane structures inside. We further characterized the ER bulbs by transmission electron microscopy (TEM). In those samples, ER was labeled by an Apex 2 chimera and stained with diaminobenzidine (DAB) [35]. In dga1 $\Delta$ lro1 $\Delta$ cells, we observed membrane-like electron-dense structures on or connected to the ER (Fig. 2d). The electron-dense structures were absent in wild-type cells, implying that they correspond to the bulbous structures seen under light microscopy. In time-lapse imaging, the bulbous structures appeared to emerge from the ER (Fig. 2e). For Golgi proteins that go back to the ER, their translocation to the regular ER network (both nuclear and peripheral pool) occurred first, in a time period that corresponds to that of ER bulb emergence (Fig. 2e). The appearance of Golgi proteins in the ER bulbs occurred much later, close to $1 \mathrm{~h}$ after starvation (Fig. 2e), which implies that the presence of Golgi proteins at the bulbs is secondary to their regression into the ER. These results demonstrate that the impact of blocking TAG synthesis is not limited to the autophagy pathway. It led to significant disturbances in the endomembrane system, disrupting normal ER-Golgi protein trafficking.

We previously reported that the autophagy defect in dga1 1 lro1 $\Delta$ cells was characterized by reduced recruitment of Atg8, Atg1, and Atg5, but not the scaffold proteins, to the phagophore assembly site (PAS) [26]. To examine the relationship between endomembrane alterations and autophagy, we experimented with blocking ER protein export using a temperature-sensitive mutant of SEC16. Upon shifting to non-permissive temperature, sec16-ts cells gradually lost punctate distribution of Golgi marker proteins (Fig. 1e, f), partially reproducing

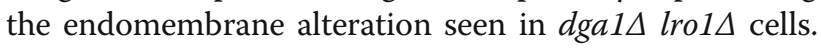
Inactivation of Sec16 also reduced PAS recruitment of Atg8 and Atg1 (Fig. 1e, g). This result is consistent with ER-Golgi trafficking defect being a major contributor to

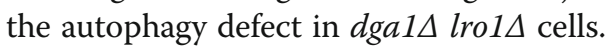

\section{Endomembrane defects in TAG production defective cells are caused by the accumulation of an intermediate metabolite}

Regardless of the complexity of potential downstream signaling, the effect of blocking a biochemical reaction 

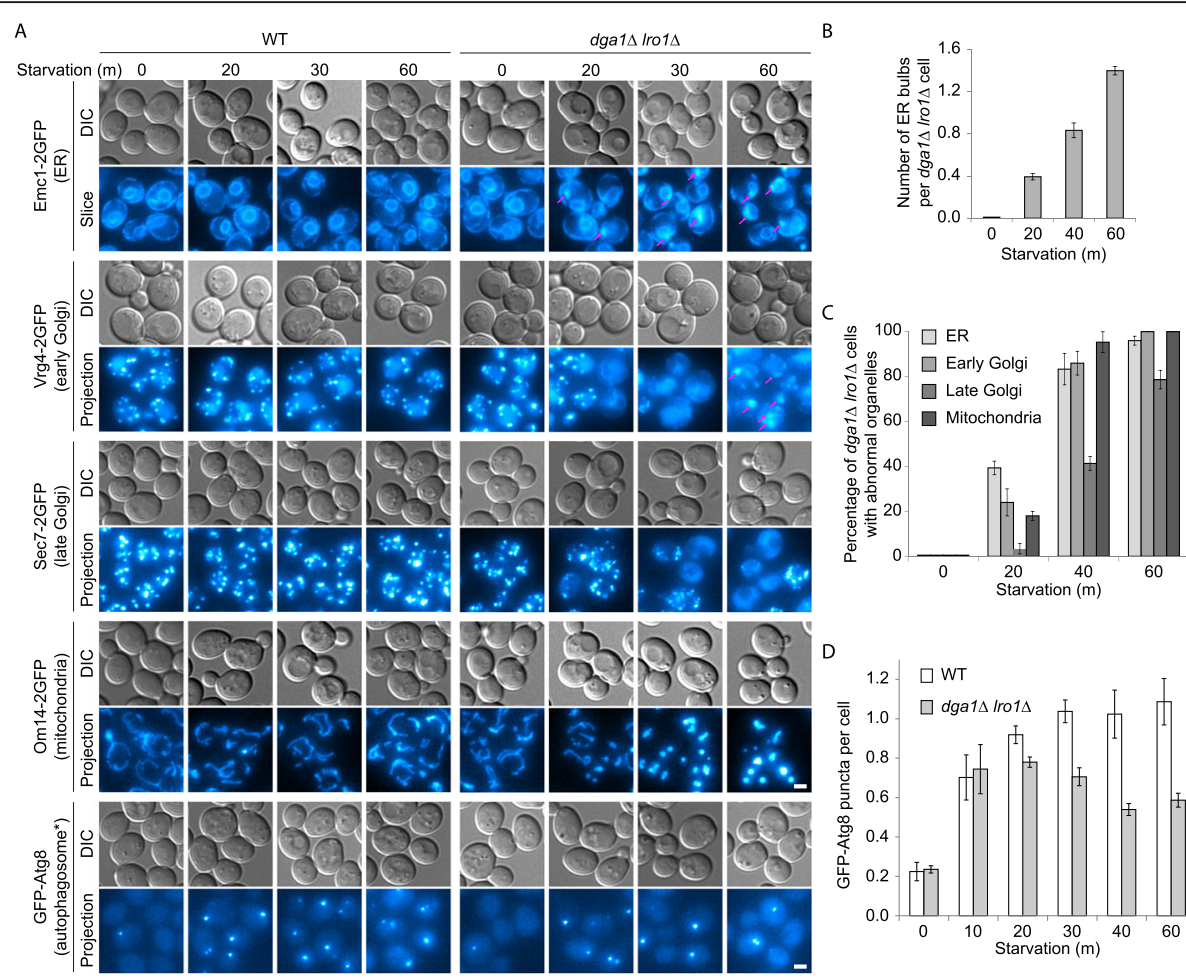

E
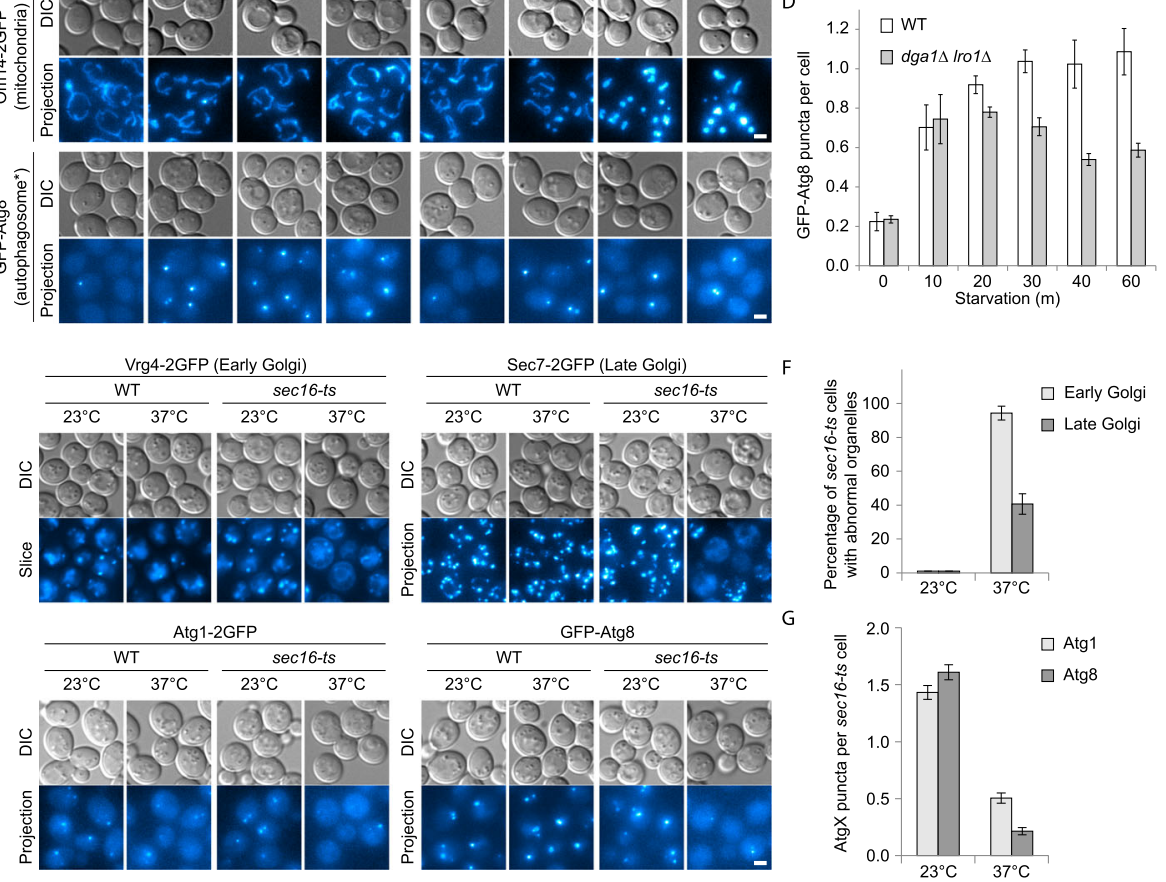

Fig. 1 Block in TAG synthesis disrupts the endomembrane system. a Starvation triggers alterations in the ER, Golgi, and mitochondrial morphology in cells defective in TAG synthesis $($ dgal $\Delta$ /ro1 $\Delta$ ). Cells expressing indicated organelle markers were transferred from rich medium to nitrogen starvation medium. Organelle morphology was observed by fluorescent microscopy at the indicated time points. Representative images from three independent repeats are shown. DIC, differential interference contrast; Slice, a single slice in the fluorescence z-stack; Projection, max intensity projection of the fluorescence z-stack. Autophagosome*, complete or incomplete autophagosomal structure. Arrows, bulbous structures on the ER. Scale bar, 2 um. b-d Quantification of organelle defects in $\mathbf{a}$. $\mathbf{b}$ Number of ER bulbs per cell. c Percentage of cells displaying abnormal organelle morphology (ER bulb formation, Golgi disappearance, mitochondrial fragmentation). d Progression of autophagy defect as indicated by the decline in the number of GFP-Atg8 dots. Error bar, standard deviation, $n=3$. e Inhibition of ER exit leads to disappearance of Golgi and impairment of Atg protein recruitment. sec16-ts cells expressing indicated organelle markers were first grew to mid-log phase under permissive temperature, then transferred to nitrogen starvation medium and incubated under either permissive temperature or non-permissive temperature for $1 \mathrm{~h}$. Images presented as in a. f Quantification of Golgi defects in e. Error bar, standard deviation, $n=3$. g Quantification of Atg1 and Atg8 recruitment defects in e. Error bar, standard deviation, $n=3$

often stems from alterations in the levels of its upstream metabolites, downstream metabolites, or a combination thereof. Here, we took a stepwise approach to pinpoint the critical metabolite causing the endomembrane and autophagy disruptions. First, we analyzed mutants lacking enzymes potentially involved in TAG hydrolysis or product utilization [32] and found no substantial alterations in autophagic flux, as measured by the pho8 $\Delta 60$ assay and GFP-Atg8 processing assay (Additional file 1: Fig. S1B-C). We and others have previously 

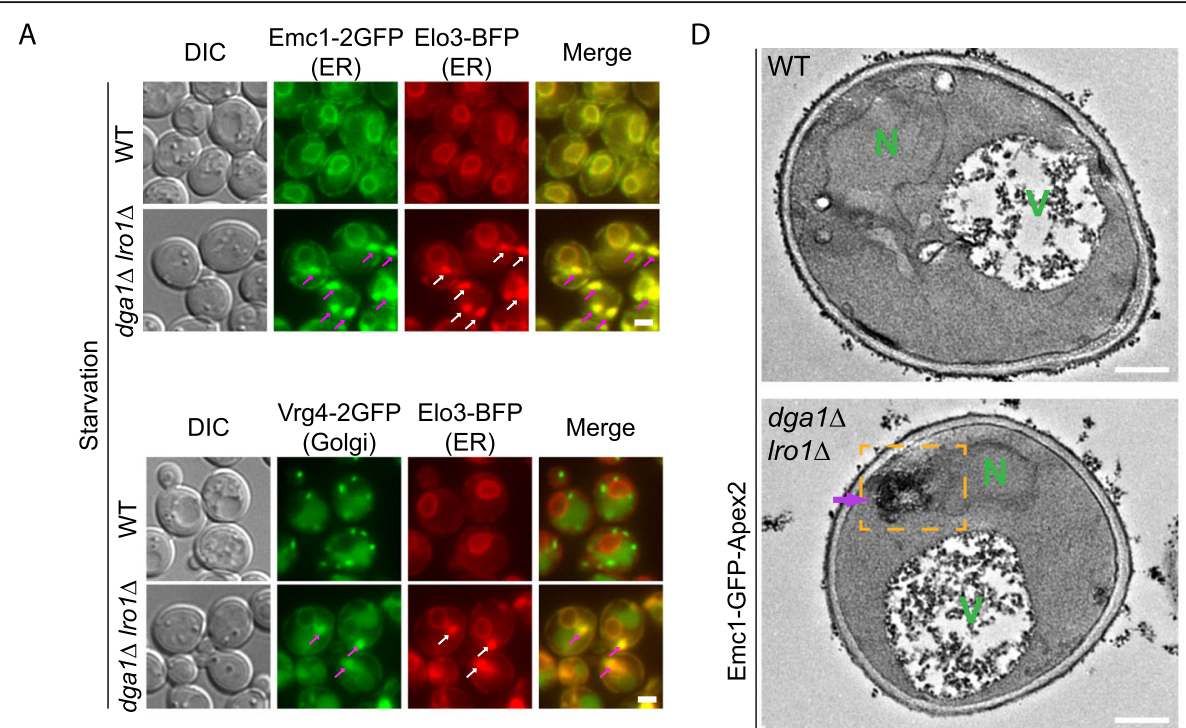

B
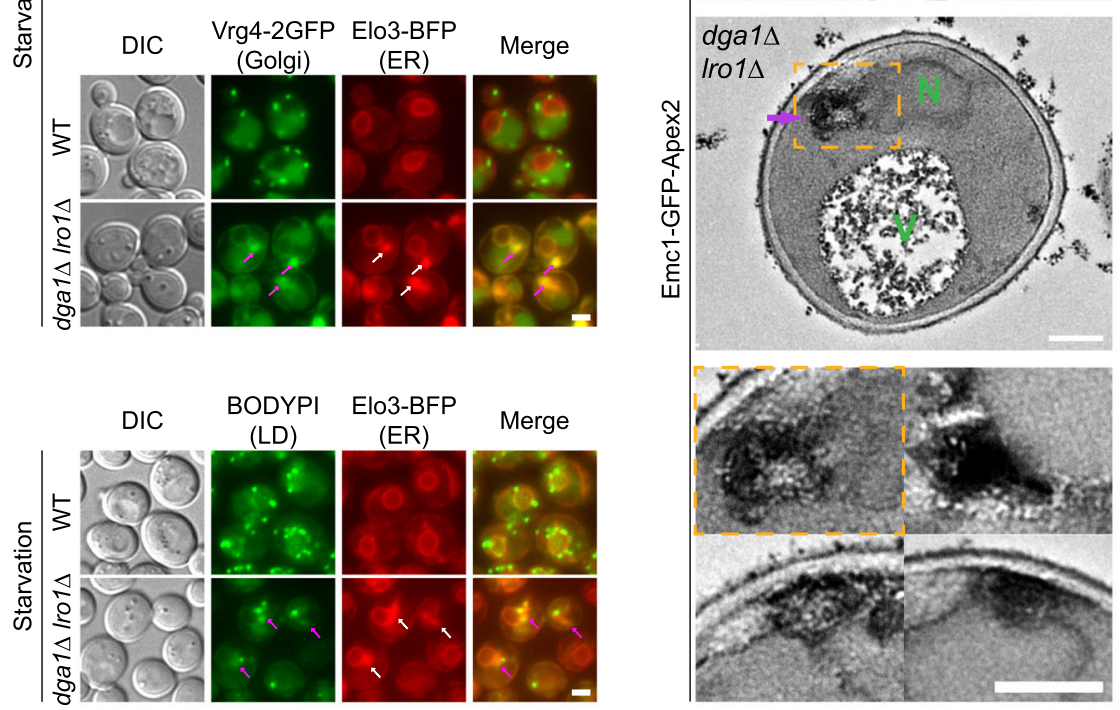

C

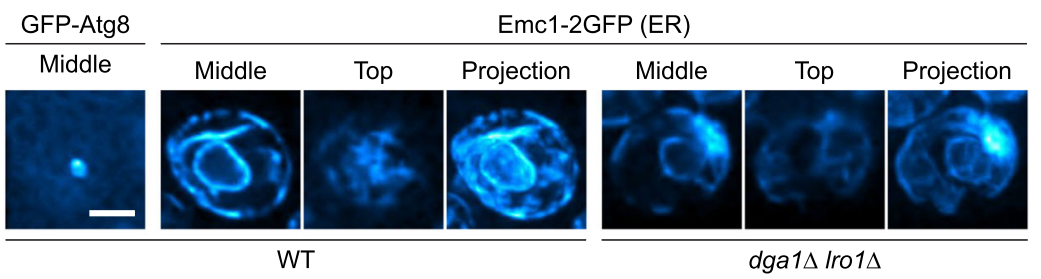

E

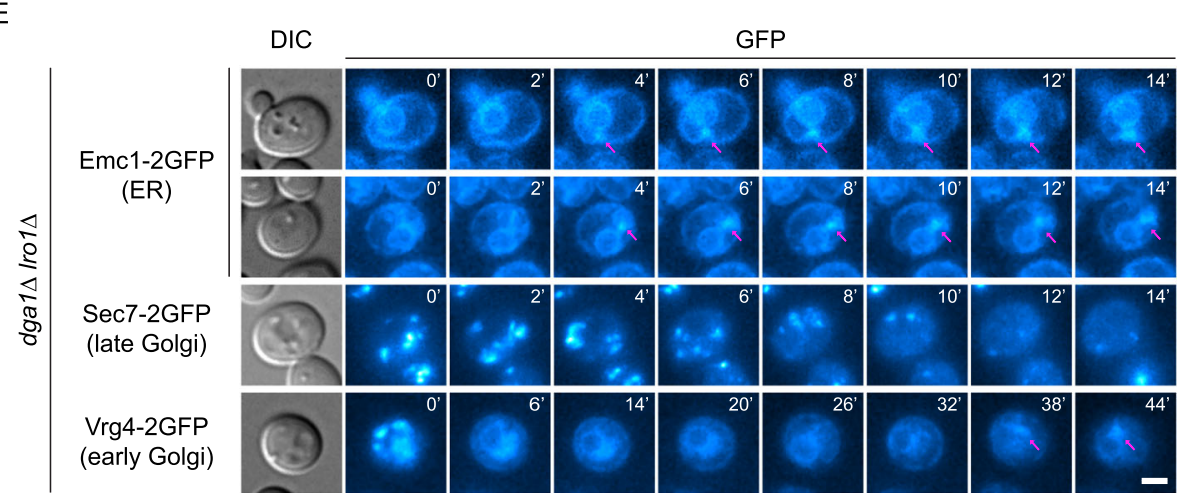

Fig. 2 (See legend on next page.) 


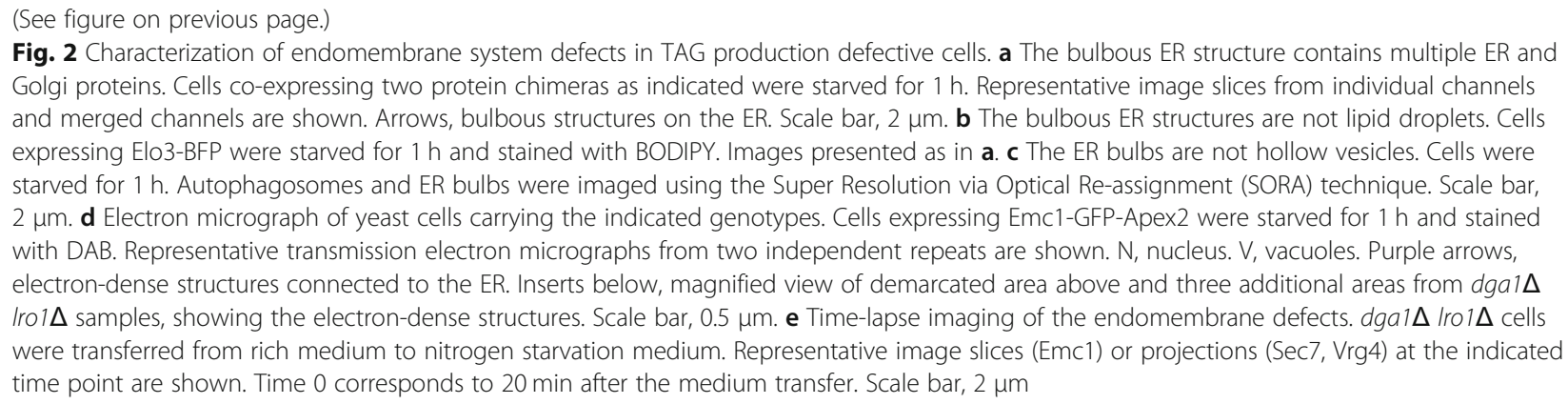

shown that the well-established TAG lipases, Tgl3 and Tgl4, are not essential for autophagy [26, 32]. Concluding that autophagy does not require TAG utilization, we focused our remaining analysis on upstream reactions leading to or diverting from TAG synthesis.

TAG and phospholipids are both glycerolipids and share early stages of their biosynthesis up to phosphatidic acid (PA) and diacylglycerol (DAG) (Additional file 1: Fig. S1A). We found that upregulation of phospholipid synthesis, either by relieving transcriptional repression of the enzymes (opi1 $\Delta$ ) or by addition of reactants (inositol, choline, and ethanolamine/ICE) [31], led to restoration of Golgi and mitochondria morphology (Fig. 3a, b). The ER morphology was largely restored with only sporadic bulbous structures, but with a visible expansion of nonnuclear ER membranes (Fig. 3a, b). Such expansion has been associated with upregulation of phospholipid biosynthesis previously $[34,36]$. Among the reactants tested, choline alone was able to ameliorate the defects significantly (data not shown); however, the addition of all three (ICE) led to maximal recovery. Autophagy in dga $1 \Delta$ lro1 $1 \Delta$ was also recovered by opi1 $\Delta$ and ICE supplementation, as indicated by the formation of GFPAtg8 puncta, GFP-Atg8 processing, and pho8 $\Delta 60$ enzymatic activation (Fig. 4a, b, e-g). Furthermore, the characteristic Atg1 and Atg5 recruitment defect was reverted by the addition of ICE (Fig. 4c, d).

Next, we took several approaches to decrease carbon influx towards glycerolipid synthesis, including (1) 100-fold reduction of glucose supply ( $0.02 \%$ glucose), (2) chemical inhibition of fatty acid synthase (cerulenin), (3) elimination of major fatty acyl-CoA synthetases (faa1 $\Delta$ faa4 $\Delta$ ), and (4) elimination of one of the two key lysoPA acyltransferases $(\operatorname{slc1\Delta })[31,37]$. With the first two approaches, the morphologies of ER, Golgi, and mitochondria in dga1 lro1 $\Delta$ cells remained normal upon starvation (Fig. 3a, b). For faa1 $\Delta$ faa $4 \Delta$, the recovery of ER morphology was partial, possibly because the recycling of free fatty acid by acyl-CoA synthetases only accounts for part of the lipid precursor influx. The partial effect caused by $\operatorname{slc} 1 \Delta$ is also consistent with the presence of remaining lysoPA acyltransferases. The absolute levels of autophagy became lower with less glucose
(Fig. 4a, b, h), possibly reflecting the reliance of autophagy on energy production. Cerulenin addition also reduced total autophagic flux (Fig. 4e, i). Nevertheless, both low glucose and cerulenin caused the levels of autophagy to become comparable between dga1 lro1 $\Delta$ and wild-type controls. The PAS recruitment of Atg1 and Atg5 was difficult to assess in low glucose condition due to dim signal (data not shown). The recruitment of these two Atg proteins became normal with cerulenin treatment (Fig. 4c, d). For faa1 $1 \Delta$ faa $4 \Delta$, autophagy was fully restored, as indicated by all three assays (Fig. 4a, b, e, j). For slc1 $\Delta$, although the number of GFP-Atg8 was recovered (Fig. 4a, b), final autophagic flux as indicated by GFP-Atg8 processing and pho8 860 was still partially compromised (Fig. 4e, j).

The approaches we took to reduce glycerolipid synthesis interfered at the steps of glucose consumption, fatty acid synthesis, free fatty acid utilization, and lyso-PA to PA conversion, respectively. With all four approaches being effective at rescuing the phenotypes, these data collectively point to metabolites downstream of PA synthesis as the endomembrane disrupter. Combined with the fact that diverting PA and DAG towards phospholipid synthesis also leads to phenotype rescue, these data imply that the defects in endomembrane system and autophagy in dga1 1 lro1 $\Delta$ cells are caused by the accumulation of an intermediate metabolite, with PA and DAG being the primary suspects.

Starvation invokes complex changes in the signaling network. To examine if the connection under investigation between lipids and the endomembrane system depends on starvation, we boosted glycerolipid synthesis in cells under nutrient-rich condition by supplementing the growth medium with oleic acid (OA). While wild-type

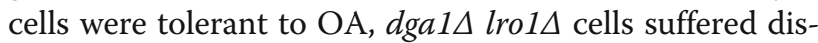
turbance in the endomembrane system (Additional file 1: Fig. S3A-B). The responses were overall similar to those under starvation, albeit with a more prominent redistribution of ER markers to the bulbs, leaving the regular ER network faintly visible (Additional file 1: Fig. S3A). Possibly because of this stronger ER response, the ER recovery brought by opi1 1 was only partial. As acylCoA synthetases are essential in the assimilation of exogenous fatty acids, OA-induced disruptions were 


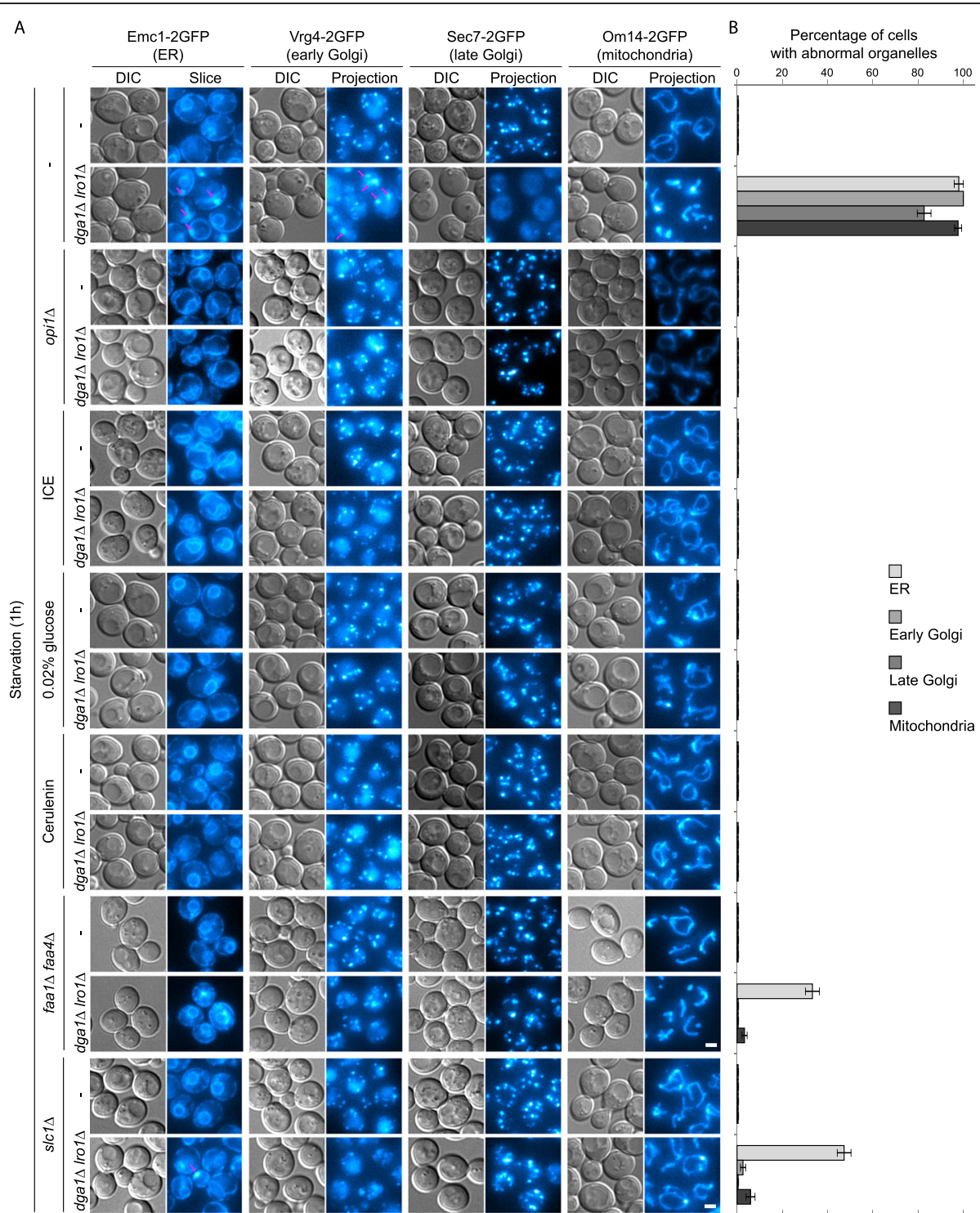

Fig. 3 Upregulating phospholipid synthesis or constraining precursor influx rescues endomembrane defects in TAG production defective cells. $\mathbf{a}$, b Phospholipid production was upregulated by (1) knocking out OPII or (2) supplying key reactants (inositol, choline, and ethanolamine/ICE). Precursor influx was constrained by (1) 100 -fold reduction of glucose supply (0.02\% glucose), (2) chemical inhibition of fatty acid synthase (cerulenin), (3) elimination of major fatty acyl-CoA synthetases (faa $1 \Delta$ faa $4 \Delta$ ), or (4) elimination of a key lysoPA acyltransferase (s/c $1 \Delta)$. a Representative images presented as in Fig. 1a. Scale bar, $2 \mu \mathrm{m}$. b Quantification of cells displaying organelle defects. Error bar, standard deviation, $n=3$

completely prevented by faa $1 \Delta$ faa $4 \Delta$. For $\operatorname{slc} 1 \Delta$, the recovery of organelle morphology was again partial. These data imply that with or without starvation, the same lipids are causing the disruptive effects.

\section{Block in TAG synthesis leads to concentration of DAG at the ER}

We then examined the subcellular localization and total

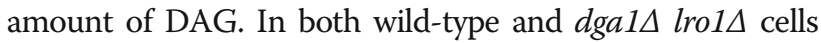
under growing condition, DAG was predominantly present at the following sites: nascent buds, vacuolar membrane, late endosomes, and late Golgi/early endosomes, as indicated by the signal of a fluorescent protein probe based on PKC $\delta$ C1 domain (GFP-PKC 8 ) (Fig. 5a, b) [38]. The signal on the buds was the brightest. No signal was discernable on the early Golgi (data not shown). Only some very faint signal of the probe was on the ER. Starvation did not change the overall distribution of DAG in wild-type cells other than reducing the number of nascent buds (Fig. 6a). In contrast, dga $1 \Delta$ lro1 $\Delta$ cells displayed a dramatic shift of the probe from the aforementioned sites to the ER (Fig. $5 \mathrm{a}$, b). The signal of the probe was concentrated on the bulbous structures, colocalizing with ER proteins therein. We also examined the distribution of DAG with a second probe 

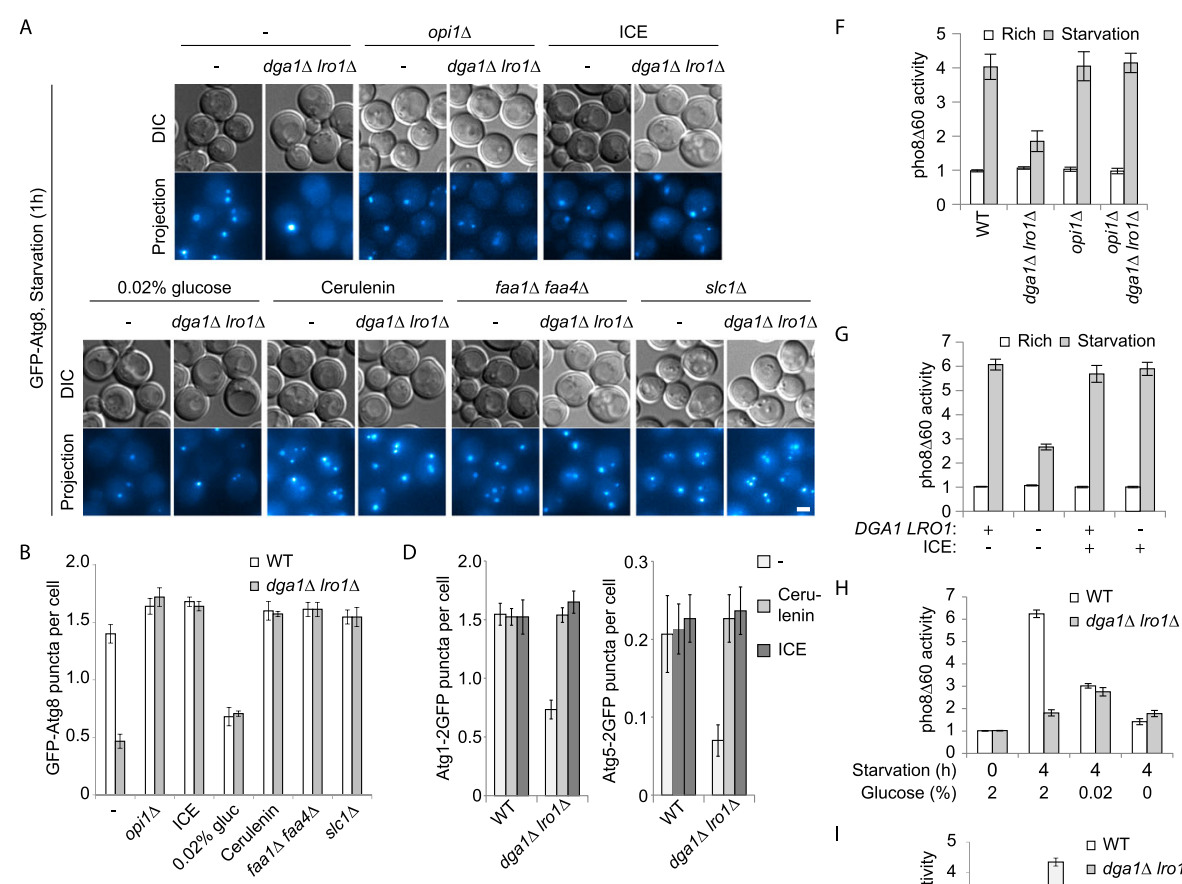

C
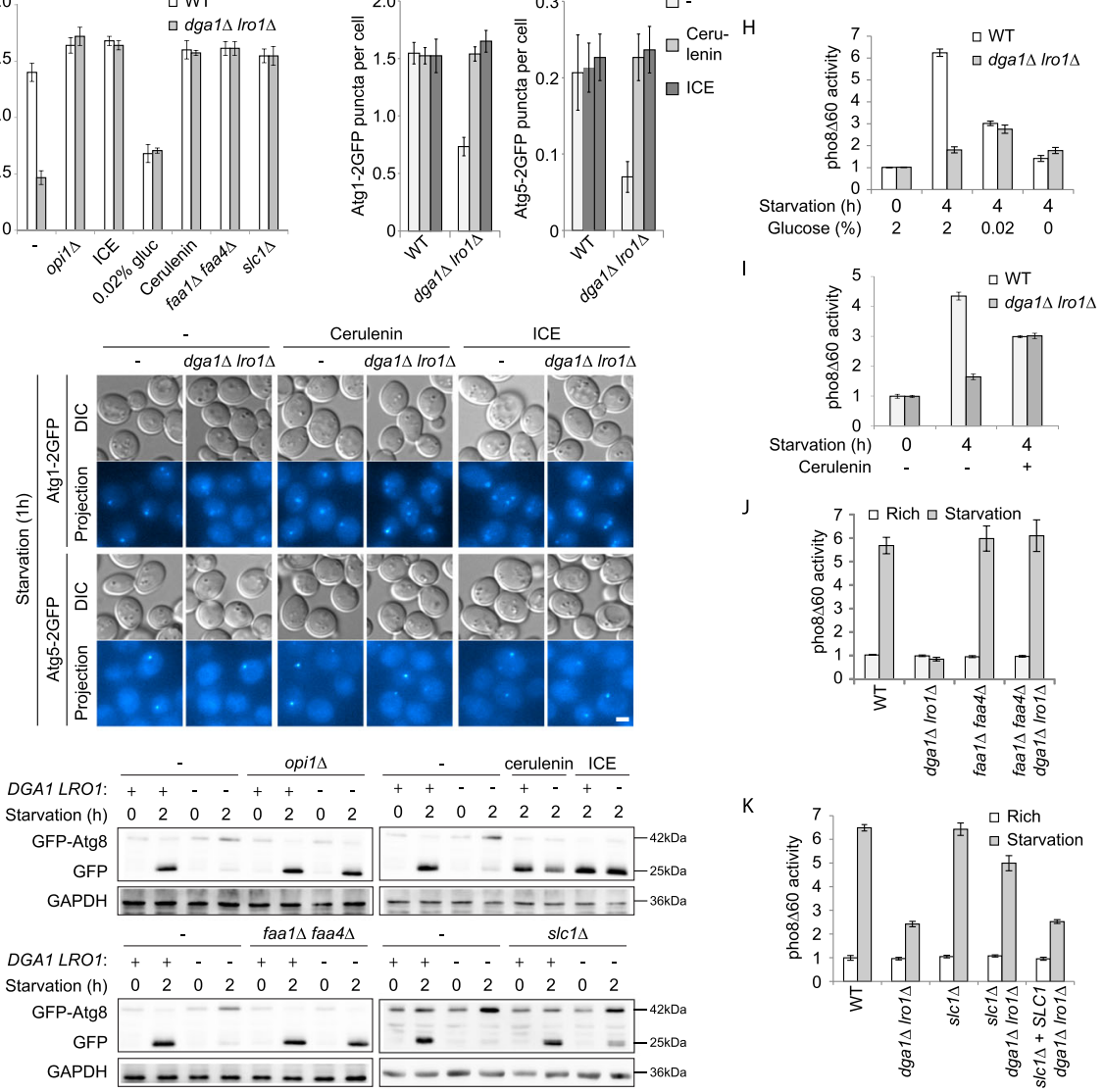

J

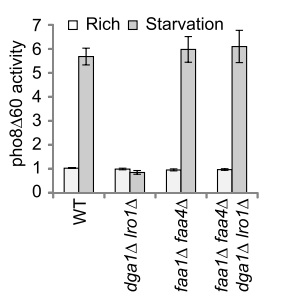

$\mathrm{K}$

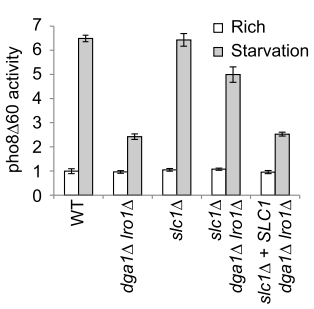

Fig. 4 Upregulating phospholipid synthesis or constraining precursor influx restores autophagy in TAG production defective cells. Autophagy was assessed by formation of GFP-Atg8 puncta $(\mathbf{a}, \mathbf{b})$, formation of Atg1 and Atg5 puncta $(\mathbf{c}, \mathbf{d})$, proteolytic processing of GFP-Atg8 (e), and pho8 $\Delta 60$ assay (f-k). Induction of phospholipid production and reduction of precursor influx were achieved as in Fig. 3. a, c Representative microscopy images presented as in Fig. 1a. Cells starved for 1 h. b, d Quantification of Atg8, Atg1, and Atg5 puncta per cell in a, c. Error bar, standard deviation, $n=3$. e Representative immunoblots from three independent repeats. Cells starved for $2 \mathrm{~h}$. $\mathbf{f}-\mathbf{k}$ pho8 860 enzymatic assay. Cells starved for $4 \mathrm{~h}$. Error bar, standard deviation, $n=3$

based on PKC $\beta$ C1 domain (GFP-PKC $\beta$ ), which displayed

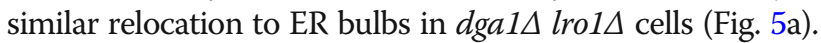
The change in subcellular DAG distribution was accompanied by an approximately threefold increase in total DAG level, as revealed by lipidomic quantification (Fig. 5c) and thin layer chromatography (TLC) (Fig. 5d). Further-

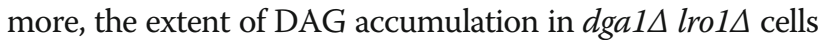

could be partially mitigated by either increased consumption (opi1 1 ) or decreased precursor supply (faa1 $1 \Delta$ $f a a 4 \Delta$, or $\operatorname{slc} 1 \Delta)$. Consistently, manipulations on intermediate consumption and precursor supply also completely (opi1 $\Delta$, ICE, $0.02 \%$ glucose, or faa $1 \Delta$ faa $4 \Delta$ ) or partially $(\operatorname{slc} 1 \Delta)$ eliminated the relocation of the DAG probe to the ER (Fig. 5e). 
A

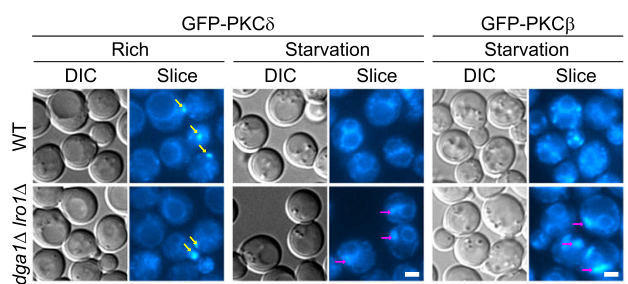

C

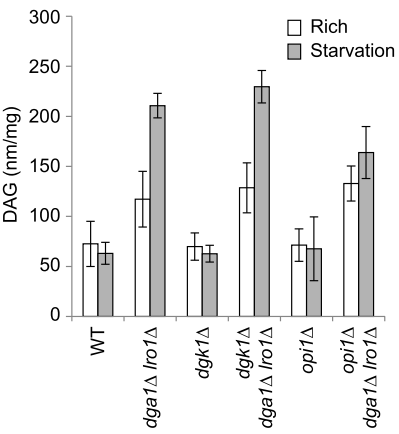

D
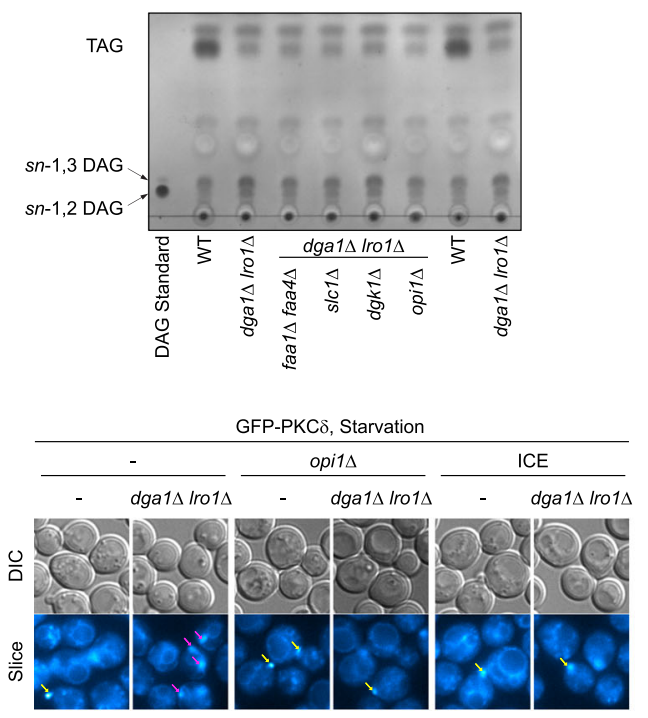

$0.02 \%$ glucose

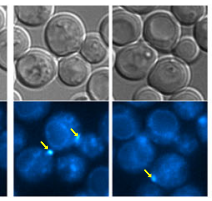

dga1 1 Iro1 $\Delta$
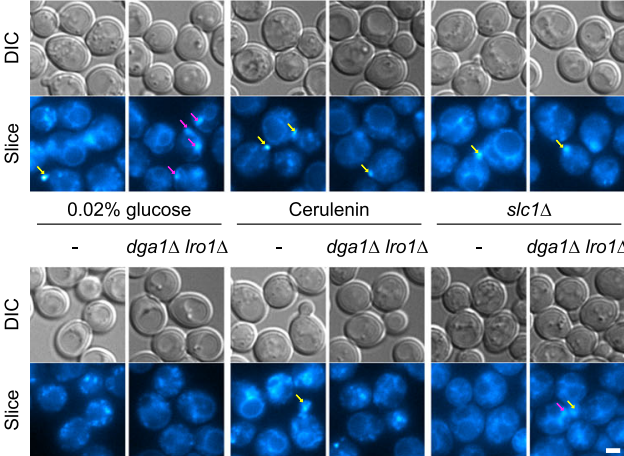

slc1s

dga1 $\operatorname{lro1\Delta }$
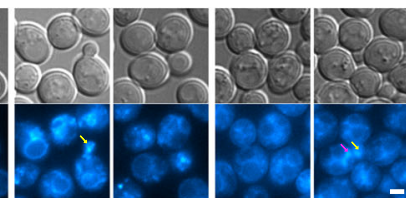

B

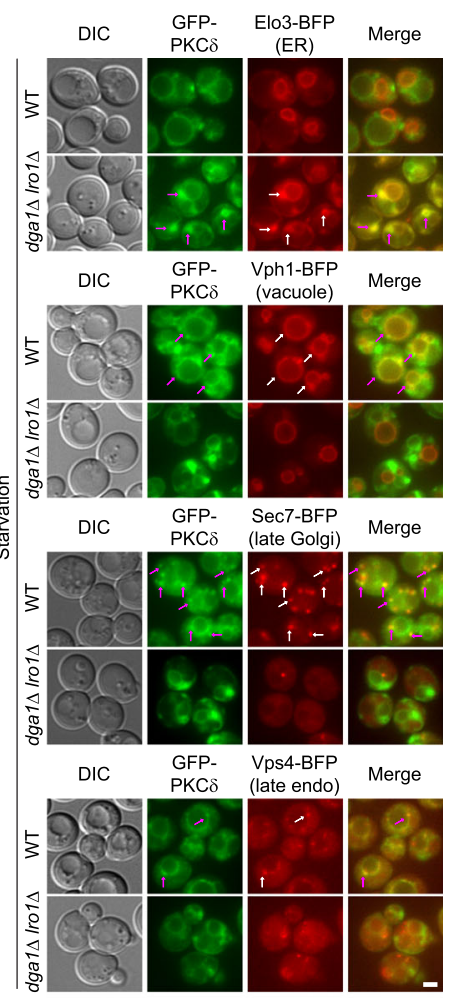

F

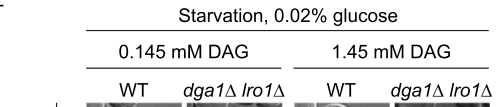

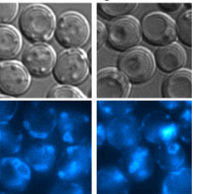
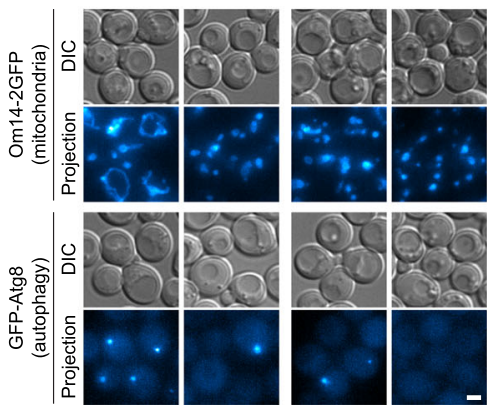

Fig. 5 Accumulation of DAG at the ER in TAG production defective cells. a Stanvation triggers intracellular DAG accumulation in dga1 $\Delta$ Iro $1 \Delta$ cells. Cells of the indicated genotype expressing DAG probes (GFP-PKC $\delta$ and GFP-PKC $\beta$ ) were grown to mid-log phase and then stanved for $1 \mathrm{~h}$. Images presented as in Fig. 1a. Yellow arrows, concentration of DAG at the buds in normal cells. Purple arrows, accumulation of DAG at intracellular bulbs. Scale bar, $2 \mu m$. $\mathbf{b}$ Starvation triggers DAG accumulation at the ER in $d g a 1 \Delta$ Irol $\Delta$ cells. Cells treated as in $\mathbf{a}$, except that additional organelle markers (ER, vacuole, late Golgi, and late endosome) were co-expressed. Image presented as in Fig. 2a. Arrows, incidences of GFP-PKC $\delta$ colocalization with organelle markers. Scale bar, $2 \mu \mathrm{m}$. c, d Total cellular DAG. Cells of the indicated genotype were grown to mid-log phase and then starved for $1 \mathrm{~h}$. Lipids were extracted and analyzed by mass spectrometry-assisted quantification (c) or thin layer chromatography (TLC) (d). c Error bar, standard deviation, $n=3$. $\mathbf{d}$ Representative image from three independent repeats. e Manipulation of glycerolipid synthesis pathway alters DAG localization. Phospholipid production was upregulated by (1) knocking out OPI1 or (2) supplying key reactants (inositol, choline, and ethanolamine/lCE). Precursor influx was constrained by (1) 100-fold reduction of glucose supply (0.02\% glucose), (2) chemical inhibition of fatty acid synthase (cerulenin), or (3) elimination of a key lysoPA acyltransferase (s/ci $\Delta$ ). Cells were starved for $1 \mathrm{~h}$. Images presented as in a. Scale bar, 2 mm. f Exogenous DAG induces endomembrane defects. 1,2-Dioctanoyl-sn-glycerol of the indicated concentrations was added to starvation medium containing $0.02 \%$ glucose. Cells were starved for $1 \mathrm{~h}$. Images presented as in Fig. 1a. Scale bar, $2 \mu \mathrm{m}$ 

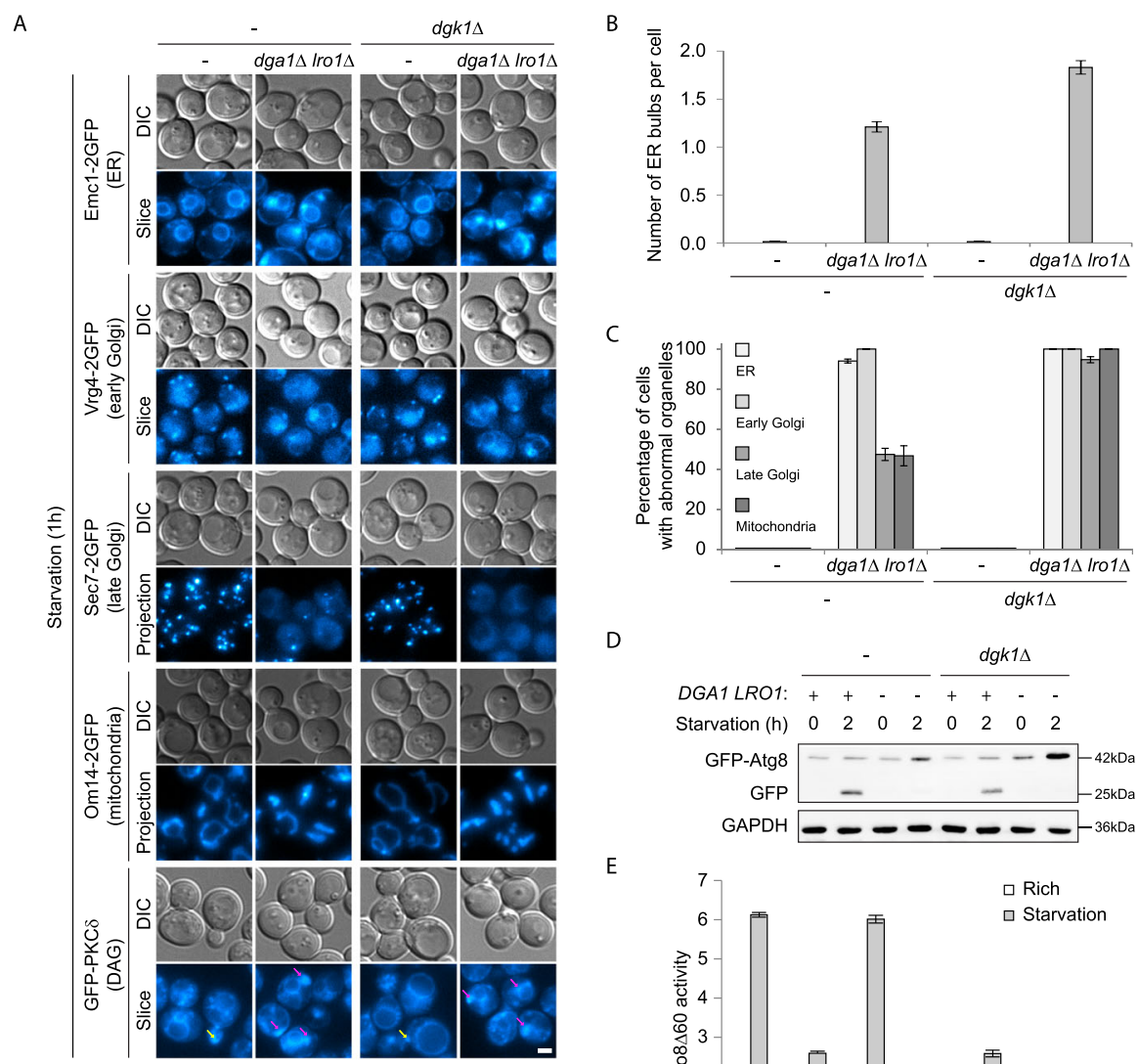

C

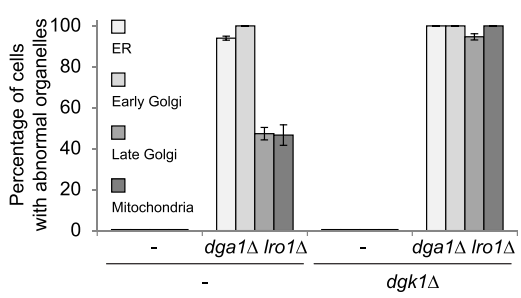

D
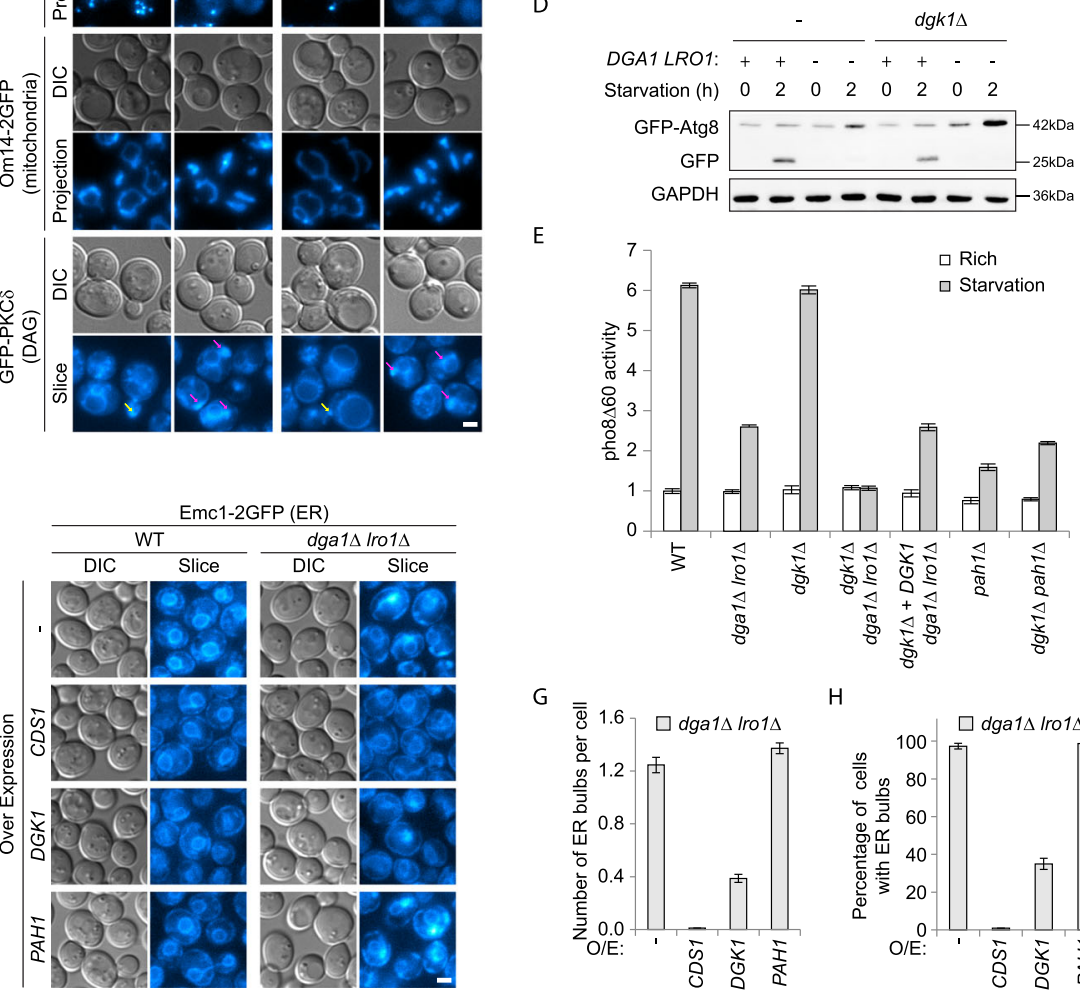

G

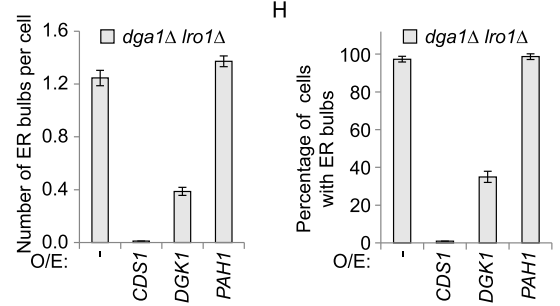

Fig. 6 Excess DAG, but not PA, is the cause of endomembrane and autophagy defects in TAG production defective cells. a-c Knocking out DGK1 aggravates endomembrane defects in dgal $\Delta$ Irol $\Delta$ cells. a Representative images presented as in Fig. 1a. Yellow arrows, concentration of DAG at the buds in normal cells. Purple arrows, ER bulbs. Scale bar, $2 \mu \mathrm{m}$. b Number of ER bulbs per cell. c Percentage of cells displaying abnormal organelle morphology (ER bulb formation, Golgi disappearance, mitochondrial fragmentation). Error bar, standard deviation, $n=3$. d, e Knocking out DGK1 aggravates autophagy defect in dga1 $\mathbf{I r o} 1 \Delta$ cells. Autophagic flux was measured by $\mathbf{d}$ proteolytic processing of GFP-Atg8, and $\mathbf{e}$ pho8 660 assay. Results presented as in Fig. 4e, f. $\mathbf{f}-\mathbf{h}$ Overexpression of DGK1 and PAH1 produces opposite effects on ER bulb formation in dga $1 \Delta$ Iro $1 \Delta$ cells. $\mathbf{f}$ Representative images presented as in Fig. 1a. Scale bar, $2 \mu \mathrm{m}$. $\mathbf{g}$ Number of ER bulbs per cell. $\mathbf{h}$ Percentage of cells displaying ER bulbs. Error bar, standard deviation, $n=3$

\section{Excess DAG, not PA, causes endomembrane defects in} TAG production defective cells

Consistent with DAG accumulation being a candidate causal factor for the disruptions, we found that the addition of 1,2-dioctanoyl-sn-glycerol (a cell permeable DAG analog) led to ER bulb formation, mitochondria

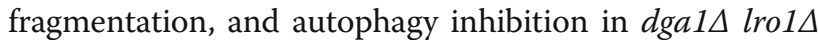
cells incubated in starvation medium containing $0.02 \%$ glucose (Fig. 5f).

The interconversion between PA and DAG is mainly mediated by two enzymes, Pah1 (PA phosphatase) and Dgk1 (DAG kinase) (Additional file 1: Fig. S1A) [31, 39, 
40]. Deletion of PAH1 also caused pleiotropic disturbances in the endomembrane system (Additional file 1: Fig. S4A). The nuclear ER/nuclear envelope was substantially enlarged in pah1s cells [41]. However, no bulbous structures were present. Both early Golgi and late Golgi/early endosome remained visible as multiple puncta, except that a fraction of early Golgi proteins also appeared on large sheet-like structures. Vacuoles became fragmented, as reported previously [42]. Instead of fragmenting into multiple spheres like in dga1 $\Delta$ lro1 $\Delta$ cells, mitochondria in pah1s cells became somewhat clustered, and the remaining tubular structures were shorter and less interconnected. Except for vacuolar fragmentation, all the changes described above were already present in pah1s cells under growing condition (Additional file 1: Fig. S4A). The changes became more severe as pah1 $\Delta$ cells entered starvation. Note that autophagy was also inhibited in pah1 1 cells, yet OPI1 knockout did not relieve the inhibition (Additional file 1: Fig. S4B). As the alterations of the endomembrane system in pah1 $\Delta$ cells were drastically different from that in dga1 $\operatorname{lro} 1 \Delta$ cells, the underlying causes of their autophagy inhibition are likely distinct. In de novo glycerolipid synthesis, PAto-DAG conversion is positioned immediate upstream of DAG-to-TAG conversion [31]. Accordingly, eliminating enzymes in both steps (pah1 lro1 $\triangle P_{G A L}$-DGA1 in glucose containing media) produced a phenotype mimicking that of pah1 (Additional file 1: Fig. S4C).

Deletion of DGK1 alone did not generate any obvious phenotype. On the other hand, deletion of DGK1 in the dga1 $\operatorname{lro} 1 \Delta$ background exaggerated the morphological changes, with ER bulb formation, Golgi disappearance, and mitochondria fragmentation all being more severe

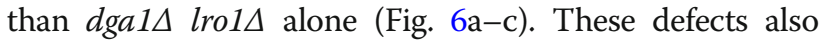
showed up sooner in the triple knockout cells (data not

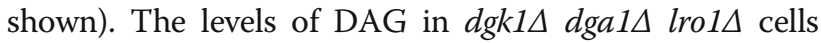
were slightly higher than those in $d g a 1 \Delta$ lro1 $\Delta$ cells, although the differences were not statistically significant (Fig. 5c). Autophagic flux in $d g k 1 \Delta d g a 1 \Delta$ lro1 $1 \Delta$ cells was

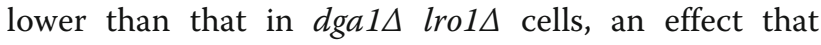
could be complemented by re-expression of DGK1 (Fig. 6d, e). In contrast, knocking out DGK1 slightly enhanced autophagy in pah1 $1 \Delta$ cells (Fig. 6e). These

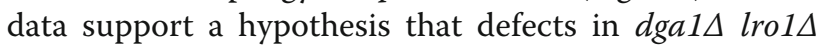
cells and pah1 $1 \Delta$ cells are each caused by the accumulation of DAG and PA, which $d g k 1 \Delta$ aggravates and alleviates, respectively.

Consistent with excess DAG being the endomembrane disruptor, overexpression of $D G K 1$ led to partial restoration of ER morphology, whereas overexpression of $P A H 1$ led to slight enhancement in ER bulb formation in dga1 $\triangle$ lro1 14 cells (Fig. 6f-h). A more robust restoration was observed with CDS1 overexpression, which diverts glycerolipid synthesis pathway towards phospholipids.

\section{ER pool of DAG is distinct from those generated at the Golgi in sphingolipid synthesis}

Considering the drastic effect of DAG on the maintenance of the Golgi, we explored whether the Golgi pool of DAG may contribute to the endomembrane disruption in cells. This pool of DAG is generated from sphingolipid biosynthesis $[43,44]$ and has been proposed to promote vesicular trafficking out of the Golgi [45].

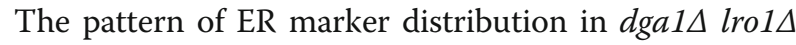
cells was unaffected by the depletion of Aur1, the first enzyme in this set of reactions (Additional file 1: Fig. S5A) [46]. Addition of an Aur1 inhibitor, aureobasidin A (AbA), up to lethal doses to $d g a 1 \Delta$ lro1 $\Delta$ cells did not affect their respective ER morphologies (Additional file 1: Fig. S5B). Myriocin, an inhibitor of serine-palmitoyl transferase (SPT) responsible for the first step in sphingolipid synthesis, did not prevent ER bulb formation either (Additional file 1: Fig. S5B). These data indicate that DAG generated from sphingolipid metabolism does not have a substantial role in the disruption of endomembrane system in dga1 1 lro1 $\Delta$ cells. We suspect that the amount of DAG from this source is insignificant or that there is a transportation barrier preventing it from joining the ER pool.

\section{Effects of intracellular DAG do not depend on Pkc1 or the unfolded protein response}

One potential downstream target of DAG is $\mathrm{C} 1$ domain containing proteins, including members of the protein kinase C (PKC) family $[47,48]$. Pkc1 is both the only $\mathrm{PKC}$ and the only $\mathrm{C} 1$ domain containing protein in yeast. Whether Pkc1 is regulated by DAG has been controversial in existing literature. We found that the subcellular localization of Pkc1 differed from that of GFPPKC $\delta$. As reported [49], Pkc1 was primarily concentrated at the nascent buds and bud necks (Additional file 1: Fig. S6A). The pattern of Pkc1 localization in dga1A lro1 $1 \Delta$ cells was comparable to that in wild-type cells under both growing and starvation conditions (Additional file 1: Fig. S6A). In addition, we deleted genes mediating Pkc1 downstream signaling (BCK1, SLT2, $M K K 1$, and $M K K 2$ ) in dga1 1 lro1 $\triangle$ cells and observed no discernable effect on the degree of ER bulb formation and mitochondria fragmentation (Additional file 1: Fig. S6B) [50]. We also investigated the potential involvement of the unfolded protein response (UPR). Treating wild-type cells with dithiothreitol (DTT) or tunicamycin triggered the UPR, as evidenced by the shift in Ire1 subcellular distribution from diffuse to punctate (Additional file 1: Fig. S6C) [51]. The same change also occurred in dga1 lro1 $\Delta$ cells upon starvation (Additional file 1: Fig. $\mathrm{S6C}$ ), indicating the presence of ER stress. However, DTT and tunicamycin were unable to induce ER bulb formation (Additional file 1: Fig. S6D). ER bulbs in cells 
lacking Dga1 and Lro1 were not reduced by knocking out IRE1 (Additional file 1: Fig. S6E), indicating that the UPR pathway is not essential for their formation. These data suggest that the effect of intracellular DAG is independent of Pkc1 signaling and the UPR and is potentially mediated by novel factors without typical $\mathrm{C} 1$ domains.

\section{Discussion}

This work began as an exploration on the connection between TAG biosynthesis and autophagy, and ended up with the identification of ER-accumulated DAG as a key molecule, the excess of which disrupts the endomembrane system (Additional file 1: Fig. S7). Starvation, like many other stress conditions, induces TAG synthesis. Under this condition, blocking the conversion of DAG to TAG causes drastic accumulation of DAG, accompanied by its redistribution from the vacuole membrane to the ER (Fig. 5). The accumulation of DAG at the ER, but not Golgi or mitochondria, also implies that the ER is the primary site of the regulatory action of DAG. This is further underscored by the fact that DAG generated at the Golgi has no significant role in the observed organelle changes (Additional file 1: Fig. S5). Disturbance of ER function may contribute to the change in mitochondrial morphology, as the ER is intimately involved in mitochondrial fission [52, 53]. The resulting relocation of Golgi marker proteins to the ER indicates that the balance of ER-Golgi trafficking pathways is severely disturbed, potentially compromising the functions of both organelles, leading to impaired Atg protein recruitment and defective autophagosome biogenesis (Figs. 1 and 2).

Our assignment of DAG as the root cause is supported by data showing that all manipulations that reduce DAG levels, either by decreasing the supply of its precursors or by increasing its consumption by phospholipid synthesis, restored the morphology of the endomembrane system and autophagy (Figs. 3 and 4). Conversely, boosting DAG levels by blocking its conversion to PA in dga1 lro1 1 cells results in even stronger disruptions to the organelles (Fig. 6). Furthermore, the effects of DAG accumulation are profoundly different from that of PA accumulation, even though both can inhibit autophagy. DAG causes the formation of ER bulbs and the regression of Golgi (Figs. 1 and 2). In contrast, PA induces the expansion of the nuclear ER and the fragmentation of the vacuole (Additional file 1: Fig. S4). Lastly, the assignment of DAG as the causal factor is fully consistent with data independently published by other colleagues [34]. Velazquez et al. previously hypothesized that autophagy

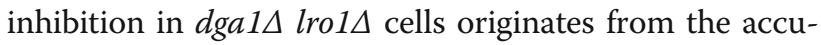
mulation of FA. Indeed, we found that inhibition of fatty acid synthase by cerulenin rescued autophagy. However, we also found that cerulenin effectively prevented ER accumulation of DAG (Fig. 5). Moreover, we show that elimination of fatty acyl-CoA synthetases (faa1 $\Delta$ faa $4 \Delta$ ), which decreases DAG by blocking FA utilization (Additional file 1: Fig. S1A), led to recovery of organelle morphology and autophagy (Figs. 3 and 4). These data indicate that FA per se is not the autophagy-inhibitory molecule and that the rescuing effect of cerulenin is instead mediated by changes in DAG.

DAG is both an intermediate metabolite and a signaling molecule. In the second role, it is best known as a second messenger generated from hydrolysis of phospholipids at the PM $[48,54]$. DAG binding proteins, including $\mathrm{C} 1$ domain containing proteins, then mediate the downstream signaling [47]. In addition to the PM, DAG is also known to function at intracellular sites. In mammalian cells, DAG is mainly present at the Golgi, ER, and nuclear envelop (NE) [55]. At the Golgi, DAG promotes the formation and/or fission of coated vesicles, in which protein kinase D (PKD) acts as a downstream target [56-59]. Furthermore, Golgi DAG participates in RAS signaling and the regulation of ER morphology [60, 61]. Upon bacterial invasion, DAG labels bacterial containing phagosomes and promotes antibacterial autophagy through activation of PKC $\delta$ [62]. In sea urchin oocytes, manipulation of DAG regulates the conversion between ER sheets and tubules [63]. Evidence of intracellular functions of DAG has also been documented in yeast, including vesicular trafficking from the Golgi and homotypic vacuole fusion, albeit direct protein targets of DAG are unknown $[45,64,65]$. Collectively, these studies demonstrate the critical need of DAG in a variety of cellular functions at both the PM and intracellular membranes.

Conversely, an oversupply of DAG may produce detrimental effects, which is the topic of the present study. Notably, DAG accumulation triggers cell death in both fission yeast and budding yeast $[8,66]$. Although the issue of subcellular localization was not directly examined, both studies employed double mutants of DGA1 and LRO1 or their homologs, similar to our study, indicating that ER may also be the primary site of DAG action in cell death pathways. ER morphological abnormalities have also been observed in dga1s lro $1 \Delta$ are $1 \Delta$ are $2 \Delta$ cells [34, 67, 68], which are defective in the synthesis of both TAG and sterol esters. The precise outcomes of ER morphology in dga $1 \Delta$ lro $1 \Delta$ are $1 \Delta$ are $2 \Delta$ cells appear to vary, possibly reflecting differences in experimental conditions as well as contributions from other lipid species. Nevertheless, all studies demonstrate a strong link between storage lipid metabolism and ER homeostasis. In particular, ER membrane aggregation in post-diauxic dga1 $\operatorname{lro} 1 \Delta$ are $1 \Delta$ are $2 \Delta$ cells was exaggerated by $d g k 1 \Delta$ [67], again consistent with DAG being the causal factor in this particular type of organelle alteration. Excess DAG's disruptive role 
in ER is potentially conserved in animals. In adipose tissue-specific DGAT1 knockout mice, high-fat diet led to significant upregulation of unfolded protein response [69]. In human adipose tissue samples, there is also a negative correlation between the expression of DGAT1 and ER stress genes [70]. In most aforementioned studies, excess DAG is generated at the ER during the biosynthesis of glycerolipids. As a result, this pool of DAG is controlled by the balance of lipid metabolism and may act chronically, producing a long-term health risk.

At present, it is not clear how excess DAG triggers the disruption of the ER and Golgi. It is possible that the effects are mediated by one or more novel downstream proteins or by direct modification of the biophysical properties of cellular membranes [71, 72]. DAG is a cone-shaped glycerolipid with a small head, the hydroxyl group. Thus, its presence in membrane favors negative spontaneous curvature. Furthermore, it is capable of fast flip-flop across lipid bilayers, which over time may negate existing membrane curvature. Both vesicle budding and fusion involve stage-specific and location-specific alterations in membrane curvature [72]. From this perspective, the concentration of DAG in membrane needs to be strictly controlled to ensure normal vesicular trafficking. At high DAG molar ratios, phase separation occurs and lens-like domain consisting primarily of DAG may form within lipid bilayers [71, 73]. The concentration of DAG in total yeast lipid is approximately $4 \%$ [74]. If the concentration of DAG in our samples is comparable with those reported, a threefold increase would put it dangerously close to the threshold of phase separation in theoretical simulations. The local concentration in ER can be even higher. The lens formation is analogous to the behavior of TAG in a proposed model of lipid droplet formation [1]. We checked ER bulb formation in two mutants of lipid droplet homeostasis, fld $1 \Delta$ and ice $2 \Delta$, but saw no obvious impact (Additional file 1: Fig. S6E). This does not rule out the possibility that other protein factors are actively involved in ER bulb formation, however. Further research is needed to elucidate the underlying mechanism.

\section{Conclusions}

In summary, this work identifies DAG as the critical lipid molecule responsible for autophagy inhibition under condition of defective TAG synthesis. Our data further implicate the disruption in ER and Golgi homeostasis as a major driving force behind observed autophagy impairments, which is in turn caused by the excess accumulation of DAG at the ER.

\section{Methods}

\section{Plasmids and strains}

Strains and plasmids constructed in this study are listed in Additional file 2: Tables S1 \& S2. Plasmids for additional organelle markers were described previously [75, 76]. Restriction sites and PCR primers utilized in plasmid construction are listed in Additional file 2: Table S3. Linearization sites and loci for genome integration of plasmids are also listed in Additional file 2: Table S2. DNA sequences of the primers used in plasmid construction are listed in Additional file 2: Table S3. Gene knockouts were performed using common homologous recombination-based technique $[77,78]$.

\section{Culturing of yeast cells}

Yeast cells in liquid culture were incubated at $30^{\circ} \mathrm{C}$ with shaking. Media recipes for YPD, SD-N, and SMD amino acid dropouts were described previously [76, 79]. Unless otherwise noted, YPD was used to grow cells to mid-log phase and SD-N was used for nitrogen starvation. When indicated, the following chemicals were added to culture media at the designated final concentrations: cerulenin $(10 \mu \mathrm{g} / \mathrm{ml}), 1,2$-dioctanoyl-sn-glycerol $(0.145 \mathrm{mM}, 1.45$ $\mathrm{mM}$ ), ICE (inositol, $0.05 \mathrm{mM}$; choline, $1 \mathrm{mM}$; ethanolamine, $1 \mathrm{mM})$, oleic acid $(0.5 \mathrm{mM})$, myriocin $(10 \mu \mathrm{g} / \mathrm{ml})$, and aureobasidin A (AbA, $1 \mu \mathrm{g} / \mathrm{ml})$.

For experiments involving GAL promoter-driven constructs, yeast strains were first re-streaked on YPGal plates. Strains were then inoculated in YPD and grown overnight to mid-log phase before shifting to SD-N medium. For experiments involving $P_{G A L}-A U R 1$, the expression of Aur1-2GFP was monitored by fluorescent microscopy once per hour. Cells were shifted to SD-N medium once the GFP signal became undetectable.

\section{TEM}

Yeast cells were first fixed in PHEM buffer $(20 \mathrm{mM}$ PIPE $\mathrm{S}, 50 \mathrm{mM}$ HEPES, $20 \mathrm{mM}$ EGTA, $4 \mathrm{mM} \mathrm{MgCl}_{2}$, adjust the $\mathrm{PH}$ to 6.9 ) containing $2 \%$ formaldehyde and $0.2 \%$ glutaraldehyde for $3 \mathrm{~h}$ at room temperature and washed three times in PHEM buffer. Cells were then incubated in DPBS (Dulbecco's phosphate-buffered saline) buffer containing $1.4 \mathrm{mM} \mathrm{DAB}$ and $0.5 \mathrm{mM} \mathrm{H}_{2} \mathrm{O}_{2}$ and kept in darkness at room temperature for $20 \mathrm{~min}$, after which cells were washed three times in DPBS. Afterwards, cells were fixed a second time in $2 \% \mathrm{KMnO}_{4}$ and kept at $4{ }^{\circ} \mathrm{C}$ for $3 \mathrm{~h}$. Cells were then washed with water several times till supernatant became colorless.

Subsequently, samples were dehydrated using graded series of ethanol $(30 \%, 50 \%, 70 \%, 80 \%, 90 \%$, then $100 \%$ three times), infiltrated with LR White resin (EMS) (33\%, 66\%, then $100 \%$ three times, $12 \mathrm{~h}$ per step at room temperature), and polymerized at $60^{\circ} \mathrm{C}$ for $48 \mathrm{~h}$. Samples 
were sectioned to $70 \mathrm{~nm}$ thin. Images were acquired on a transmission electron microscope.

\section{Total lipid extraction}

Total lipids were extracted using a modified protocol of Zhu et al. [80]. Yeast cells were pelleted and freezedried. Samples were then suspended in $500 \mu \mathrm{l}$ chloroform-methanol $(2: 1, \mathrm{v} / \mathrm{v})$ with glass beads and underwent $15 \mathrm{~min}$ of mechanical shearing on a beadmill. Lysates were centrifuged at $10,000 \mathrm{~g}$ for $5 \mathrm{~min}$, and the organic phase was moved to new tubes for collection. The chloroform-methanol extraction procedure was repeated five times. The organic phase collections were merged and evaporated at $60^{\circ} \mathrm{C}$.

\section{Lipid analysis by TLC}

TLC analysis of lipids was performed essentially as described in [81]. Lipid extracts were dissolved in chloroform and spotted on activated silica gel plates. Neutral lipids were separated using hexane-diethyl ether-acetic acid $(70: 30: 1 \mathrm{v} / \mathrm{v} / \mathrm{v})$ as the mobile phase. Plates were stained by a $0.03 \%$ Coomassie Brillant Blue R-250 solution containing $20 \%$ methanol for $15 \mathrm{~min}$ and de-stained by $20 \%$ methanol for $10 \mathrm{~min}$ [82].

\section{Lipid analysis by LC/MS}

For DAG, total lipids were extracted in the presence of internal standards (C19:0/C19:0 DAG) and analyzed on a TSQ Vantage mass spectrometer equipped with an electrospray ion source (ThermoFisher Scientific) and coupled to a UHPLC system with an ACQUITY ${ }^{\circ}$ UPLC HILIC column $(100 \times 2.1 \mathrm{~mm}, 1.7 \mu \mathrm{m}$, Waters $)$. The flow rate was set at $0.2 \mathrm{ml} / \mathrm{min}$, and the following mobile phase gradient was employed: 0 min $99 \% A+1 \% B, 10$ min $80 \% \mathrm{~A}+20 \% \mathrm{~B}, 11$ min $2 \% \mathrm{~A}+98 \% \mathrm{~B}$, and then held for $2 \mathrm{~min}$, with A being $\mathrm{ACN} / \mathrm{H}_{2} \mathrm{O}(95: 5, \mathrm{v} / \mathrm{v}, 10$ $\mathrm{mM}$ ammonium acetate) and $\mathrm{B}$ being $\mathrm{ACN} / \mathrm{H}_{2} \mathrm{O}$ (50:50, $\mathrm{v} / \mathrm{v}, 10 \mathrm{mM}$ ammonium acetate). Molecular species of DAG were analyzed following one-step derivatization with $N, N$-dimethylglycine as previously described [83].

\section{Others}

Fluorescence microscopy, pho $8 \Delta 60$ assay, and GFP-Atg8 processing assay were performed as described previously $[75,76,79]$. For quantification of microscopy images, the experiments were repeated at least 3 times, and a total of at least 150 cells were analyzed for each sample.

\section{Supplementary information}

Supplementary information accompanies this paper at https://doi.org/10. 1186/s12915-020-00837-w.

Additional file 1: Figure S1. TAG utilization is not essential for autophagy. Figure S2. Fluorescent microscopy of additional organelle markers. Figure S3. Intermediate accumulation is responsible for endomembrane defects in TAG production defective cells under growing condition upon oleic acid addition. Figure S4. PA accumulation leads to distinct alterations in the endomembrane system. Figure S5. ER bulb formation in TAG production defective cells does not rely on Golgi produced DAG. Figure S6. Endomembrane defects in TAG production defective cells do not rely on Pkc1 signaling or UPR. Figure S7.

Schematic depiction for the role of DAG in the endomembrane system.

Additional file 2: Table S1. Strains used in this study. Table S2. Plasmids constructed in this study. Table S3. DNA sequences of PCR primers.

Additional file 3. Raw data in excel file for all column graphs in figures and additional figures.

\section{Acknowledgements}

The authors would like to thank Dr. Guanghou Shui (Institute of Genetics and Developmental Biology, Chinese Academy of Sciences) and Dr. Lei Feng (Instrumental Analysis Center, SJTU) for technical assistance in lipid analysis; thank Ms. Ge Wang, Dr. Jing Liu, and Dr. Meng-Yu Yan (Instrumental Analysis Center, SJTU) for technical assistance in TEM; and thank Dr. Alwin Köhler (Medical University of Vienna) and Dr. Vanina Zaremberg (University of Calgary) for gifts of plasmids. The authors would also like to thank Mr. Zhou Peng and Dr. Xiaoling Miao (School of Life Sciences and Biotechnology, SJTU) for kind advice in experimental procedures, and Dr. Hongyuan Yang (University of New South Wales) and Dr. Daniel J Klionsky (University of Michigan, Ann Arbor) for critical reading of the manuscript.

\section{Authors' contributions}

Conceptualization: D.L., Q.G., and Z.X.; funding acquisition: J.Z., Q.G., and Z.X.; investigation: D.L., S-G.Y., C-W.H, Z-T.Z, and H.L.; project administration: J.Z.; supervision: Y.L., X.S., Q.G., and Z.X.; writing-original draft: D.L., Q.G., and Z.X. All authors read and approved the final manuscript.

\section{Funding}

This work was supported by the National Natural Science Foundation of China (grants 91754110, 91957104, and 31671431 to Z.X.; 31701200 to J.Z:; 91954102, 31871355, and 31671419 to Q.G.), Shanghai Municipal Education Commission (grant 2017-01-07-00-02-E00035 to Z.X.), Shanghai Municipal Science and Technology Commission (grant 18ZR1420400 to Z.X.), and Tianjin Research Program of Applied Basic and Cutting-edge Technologies (grant 18JCZDJC32300 to Q.G.).

Availability of data and materials

Raw data for column graphs are presented in Additional file 3: Raw Data.

Ethics approval and consent to participate

Not applicable.

\section{Competing interests}

The authors declare no competing financial interests.

\section{Author details}

${ }^{1}$ State Key Laboratory of Microbial Metabolism \& Joint International Research Laboratory of Metabolic and Developmental Sciences, School of Life Sciences and Biotechnology, Shanghai Jiao Tong University, \#800 Dong-Chuan Road, Shanghai 200240, People's Republic of China. ${ }^{2}$ School of Biology and Basic Medical Sciences, Soochow University, Suzhou, Jiangsu, People's Republic of China. ${ }^{3}$ College of Life Sciences, Nanjing Agricultural University, Nanjing, Jiangsu, People's Republic of China.

Received: 7 May 2020 Accepted: 24 July 2020

Published online: 28 August 2020

\section{References}

1. Gao M, Huang X, Song BL, Yang $H$. The biogenesis of lipid droplets: lipids take center stage. Prog Lipid Res. 2019;75:100989.

2. Gao Q, Goodman JM. The lipid droplet-a well-connected organelle. Front Cell Dev Biol. 2015;3:49.

3. Werstuck GH, Lentz SR, Dayal S, Hossain GS, Sood SK, Shi YY, Zhou J, Maeda N, Krisans SK, Malinow MR, et al. Homocysteine-induced endoplasmic 
reticulum stress causes dysregulation of the cholesterol and triglyceride biosynthetic pathways. J Clin Invest. 2001;107(10):1263-73.

4. Nguyen TB, Louie SM, Daniele JR, Tran Q, Dillin A, Zoncu R, Nomura DK, Olzmann JA. DGAT1-dependent lipid droplet biogenesis protects mitochondrial function during starvation-induced autophagy. Dev Cell. 2017;42(1):9-21 e25.

5. Fei W, Wang H, Fu X, Bielby C, Yang H. Conditions of endoplasmic reticulum stress stimulate lipid droplet formation in Saccharomyces cerevisiae. Biochem J. 2009;424(1):61-7.

6. Gubern A, Barcelo-Torns M, Casas J, Barneda D, Masgrau R, Picatoste F, Balsinde J, Balboa MA, Claro E. Lipid droplet biogenesis induced by stress involves triacylglycerol synthesis that depends on group VIA phospholipase A2. J Biol Chem. 2009;284(9):5697-708.

7. Han J, Kaufman RJ. The role of ER stress in lipid metabolism and lipotoxicity. J Lipid Res. 2016;57(8):1329-38.

8. Zhang Q, Chieu HK, Low CP, Zhang S, Heng CK, Yang H. Schizosaccharomyces pombe cells deficient in triacylglycerols synthesis undergo apoptosis upon entry into the stationary phase. J Biol Chem. 2003; 278(47):47145-55.

9. Listenberger LL, Han X, Lewis SE, Cases S, Farese RV Jr, Ory DS, Schaffer JE. Triglyceride accumulation protects against fatty acid-induced lipotoxicity. Proc Natl Acad Sci U S A. 2003;100(6):3077-82.

10. Kroemer $\mathrm{G}$, Marino $\mathrm{G}$, Levine B. Autophagy and the integrated stress response. Mol Cell. 2010;40(2):280-93.

11. Jaishy B, Abel ED. Lipids, lysosomes, and autophagy. J Lipid Res. 2016;57(9): 1619-35.

12. Axe EL, Walker SA, Manifava M, Chandra P, Roderick HL, Habermann A, Griffiths G, Ktistakis NT. Autophagosome formation from membrane compartments enriched in phosphatidylinositol 3-phosphate and dynamically connected to the endoplasmic reticulum. J Cell Biol. 2008; 182(4):685-701.

13. Graef M, Friedman JR, Graham C, Babu M, Nunnari J. ER exit sites are physical and functional core autophagosome biogenesis components. Mol Biol Cell. 2013;24(18):2918-31.

14. Suzuki K, Akioka M, Kondo-Kakuta C, Yamamoto H, Ohsumi Y. Fine mapping of autophagy-related proteins during autophagosome formation in Saccharomyces cerevisiae. J Cell Sci. 2013;126(Pt 11):2534-44.

15. Valverde DP, Yu S, Boggavarapu V, Kumar N, Lees JA, Walz T, Reinisch KM, Melia TJ. ATG2 transports lipids to promote autophagosome biogenesis. J Cell Biol. 2019;218(6):1787-98.

16. Osawa T, Kotani T, Kawaoka T, Hirata E, Suzuki K, Nakatogawa H, Ohsumi Y, Noda NN. Atg2 mediates direct lipid transfer between membranes for autophagosome formation. Nat Struct Mol Biol. 2019;26(4):281-8.

17. Wang J, Davis S, Menon S, Zhang J, Ding J, Cervantes S, Miller E, Jiang Y, Ferro-Novick S. Ypt1/Rab1 regulates Hrr25/CK1 delta kinase activity in ERGolgi traffic and macroautophagy. J Cell Biol. 2015;210(2):273-85.

18. Karanasios E, Walker SA, Okkenhaug H, Manifava M, Hummel E, Zimmermann H, Ahmed Q, Domart MC, Collinson L, Ktistakis NT. Autophagy initiation by ULK complex assembly on ER tubulovesicular regions marked by ATG9 vesicles. Nat Commun. 2016;7:12420.

19. Ge L, Zhang M, Kenny SJ, Liu D, Maeda M, Saito K, Mathur A, Xu K, Schekman R. Remodeling of ER-exit sites initiates a membrane supply pathway for autophagosome biogenesis. EMBO Rep. 2017;18(9):1586-603.

20. Yu L, Chen Y, Tooze SA. Autophagy pathway: cellular and molecular mechanisms. Autophagy. 2018;14(2):207-15.

21. Farhan $H$, Kundu M, Ferro-Novick $S$. The link between autophagy and secretion: a story of multitasking proteins. Mol Biol Cell. 2017;28(9):1161-4.

22. Andrejeva G, Gowan S, Lin G, Wong Te Fong AL, Shamsaei E, Parkes HG, Mui J, Raynaud Fl, Asad Y, Vizcay-Barrena G, et al. De novo phosphatidylcholine synthesis is required for autophagosome membrane formation and maintenance during autophagy. Autophagy. 2020;16(6):1044-60.

23. Nishimura $\mathrm{T}$, Tamura $\mathrm{N}$, Kono $\mathrm{N}$, Shimanaka $\mathrm{Y}$, Arai $\mathrm{H}$, Yamamoto $\mathrm{H}$, Mizushima N. Autophagosome formation is initiated at phosphatidylinositol synthase-enriched ER subdomains. EMBO J. 2017;36(12):1719-35.

24. Schutter M, Giavalisco P, Brodesser S, Graef M. Local fatty acid channeling into phospholipid synthesis drives phagophore expansion during autophagy. Cell. 2020;180(1):135-149.e14.

25. Reggiori F, Klionsky DJ. Autophagic processes in yeast: mechanism, machinery and regulation. Genetics. 2013;194(2):341-61.

26. Li D, Song JZ, Li H, Shan MH, Liang Y, Zhu J, Xie Z. Storage lipid synthesis is necessary for autophagy induced by nitrogen starvation. FEBS Lett. 2015; 589(2):269-76.
27. Cabodevilla AG, Sanchez-Caballero L, Nintou E, Boiadjieva VG, Picatoste F, Gubern A, Claro E. Cell survival during complete nutrient deprivation depends on lipid droplet-fueled beta-oxidation of fatty acids. J Biol Chem. 2013;288(39):27777-88

28. Rambold AS, Cohen S, Lippincott-Schwartz J. Fatty acid trafficking in starved cells: regulation by lipid droplet lipolysis, autophagy, and mitochondrial fusion dynamics. Dev Cell. 2015;32(6):678-92.

29. Oelkers P, Cromley D, Padamsee M, Billheimer JT, Sturley SL. The DGA1 gene determines a second triglyceride synthetic pathway in yeast. J Biol Chem. 2002;277(11):8877-81.

30. Oelkers P, Tinkelenberg A, Erdeniz N, Cromley D, Billheimer JT, Sturley SL. A lecithin cholesterol acyltransferase-like gene mediates diacylglycerol esterification in yeast. J Biol Chem. 2000;275(21):15609-12.

31. Henry SA, Kohlwein SD, Carman GM. Metabolism and regulation of glycerolipids in the yeast Saccharomyces cerevisiae. Genetics. 2012;190(2): $317-49$.

32. Shpilka T, Welter E, Borovsky N, Amar N, Mari M, Reggiori F, Elazar Z. Lipid droplets and their component triglycerides and steryl esters regulate autophagosome biogenesis. EMBO J. 2015;34(16):2117-31.

33. Regnacq M, Voisin P, Sere YY, Wan B, Soeroso VMS, Bernard M, Camougrand N, Bernard FX, Barrault C, Berges T. Increased fatty acid synthesis inhibits nitrogen starvation-induced autophagy in lipid droplet-deficient yeast. Biochem Biophys Res Commun. 2016;477(1):33-9.

34. Velazquez AP, Tatsuta T, Ghillebert R, Drescher I, Graef M. Lipid dropletmediated ER homeostasis regulates autophagy and cell survival during starvation. J Cell Biol. 2016;212(6):621-31.

35. Lam SS, Martell JD, Kamer KJ, Deerinck TJ, Ellisman MH, Mootha VK, Ting AY. Directed evolution of APEX2 for electron microscopy and proximity labeling. Nat Methods. 2015;12(1):51-4.

36. Schuck S, Prinz WA, Thorn KS, Voss C, Walter P. Membrane expansion alleviates endoplasmic reticulum stress independently of the unfolded protein response. J Cell Biol. 2009;187(4):525-36.

37. Athenstaedt K, Daum G. Biosynthesis of phosphatidic acid in lipid particles and endoplasmic reticulum of Saccharomyces cerevisiae. J Bacteriol. 1997; 179(24):7611-6.

38. Ganesan S, Shabits BN, Zaremberg V. Tracking diacylglycerol and phosphatidic acid pools in budding yeast. Lipid Insights. 2015;8(Suppl 1):75-85.

39. Han GS, Wu WI, Carman GM. The Saccharomyces cerevisiae Lipin homolog is a Mg2+-dependent phosphatidate phosphatase enzyme. J Biol Chem. 2006;281(14):9210-8.

40. Han GS, O'Hara L, Siniossoglou S, Carman GM. Characterization of the yeast DGK1-encoded CTP-dependent diacylglycerol kinase. J Biol Chem. 2008; 283(29):20443-53.

41. Santos-Rosa H, Leung J, Grimsey N, Peak-Chew S, Siniossoglou S. The yeast lipin Smp2 couples phospholipid biosynthesis to nuclear membrane growth. EMBO J. 2005;24(11):1931-41.

42. Sasser T, Qiu QS, Karunakaran S, Padolina M, Reyes A, Flood B, Smith S, Gonzales C, Fratti RA. Yeast lipin 1 orthologue pah1p regulates vacuole homeostasis and membrane fusion. J Biol Chem. 2012:287(3):2221-36.

43. Cowart LA, Obeid LM. Yeast sphingolipids: recent developments in understanding biosynthesis, regulation, and function. Biochim Biophys Acta. 2007;1771(3):421-31

44. Breslow DK. Sphingolipid homeostasis in the endoplasmic reticulum and beyond. Cold Spring Harb Perspect Biol. 2013;5(4):a013326.

45. Kearns BG, McGee TP, Mayinger P, Gedvilaite A, Phillips SE, Kagiwada S, Bankaitis VA. Essential role for diacylglycerol in protein transport from the yeast Golgi complex. Nature. 1997;387(6628):101-5.

46. Nagiec MM, Nagiec EE, Baltisberger JA, Wells GB, Lester RL, Dickson RC. Sphingolipid synthesis as a target for antifungal drugs. Complementation of the inositol phosphorylceramide synthase defect in a mutant strain of Saccharomyces cerevisiae by the AUR1 gene. J Biol Chem. 1997;272(15):9809-17.

47. Colon-Gonzalez F, Kazanietz MG. C1 domains exposed: from diacylglycerol binding to protein-protein interactions. Biochim Biophys Acta. 2006;1761(8): 827-37.

48. Newton AC, Bootman MD, Scott JD. Second messengers. Cold Spring Harb Perspect Biol. 2016;8(8):a005926. https://doi.org/10.1101/cshperspect. a005926.

49. Andrews PD, Stark MJ. Dynamic, Rho1p-dependent localization of Pkc1p to sites of polarized growth. J Cell Sci. 2000;113(Pt 15):2685-93.

50. Heinisch JJ, Rodicio R. Protein kinase C in fungi-more than just cell wall integrity. FEMS Microbiol Rev. 2018;42(1):22-39. 
51. Welihinda AA, Kaufman RJ. The unfolded protein response pathway in Saccharomyces cerevisiae. Oligomerization and trans-phosphorylation of Ire1p (Ern1p) are required for kinase activation. J Biol Chem. 1996;271(30): 18181-7.

52. Friedman JR, Lackner LL, West M, DiBenedetto JR, Nunnari J, Voeltz GK. ER tubules mark sites of mitochondrial division. Science. 2011;334(6054):358-62.

53. Korobova F, Ramabhadran V, Higgs HN. An actin-dependent step in mitochondrial fission mediated by the ER-associated formin INF2. Science. 2013;339(6118):464-7.

54. Kadamur G, Ross EM. Mammalian phospholipase C. Annu Rev Physiol. 2013; 75:127-54.

55. Domart MC, Hobday TM, Peddie CJ, Chung GH, Wang A, Yeh K, Jethwa N, Zhang Q, Wakelam MJ, Woscholski R, et al. Acute manipulation of diacylglycerol reveals roles in nuclear envelope assembly \& endoplasmic reticulum morphology. PLoS One. 2012;7(12):e51150.

56. Baron $\mathrm{CL}$, Malhotra V. Role of diacylglycerol in PKD recruitment to the TGN and protein transport to the plasma membrane. Science. 2002;295(5553): 325-8.

57. Litvak V, Dahan N, Ramachandran S, Sabanay H, Lev S. Maintenance of the diacylglycerol level in the Golgi apparatus by the Nir2 protein is critical for Golgi secretory function. Nat Cell Biol. 2005;7(3):225-34

58. Fernandez-Ulibarri I, Vilella M, Lazaro-Dieguez F, Sarri E, Martinez SE, Jimenez N, Claro E, Merida I, Burger KN, Egea G. Diacylglycerol is required for the formation of COPI vesicles in the Golgi-to-ER transport pathway. Mol Biol Cell. 2007;18(9):3250-63.

59. Asp L, Kartberg F, Fernandez-Rodriguez J, Smedh M, Elsner M, Laporte F, Barcena M, Jansen KA, Valentijn JA, Koster AJ, et al. Early stages of Golgi vesicle and tubule formation require diacylglycerol. Mol Biol Cell. 2009;20(3): 780-90

60. Bivona TG, Perez De Castro I, Ahearn IM, Grana TM, Chiu VK, Lockyer PJ, Cullen PJ, Pellicer A, Cox AD, Philips MR. Phospholipase Cgamma activates Ras on the Golgi apparatus by means of RasGRP1. Nature. 2003;424(6949): 694-8.

61. Roose JP, Mollenauer M, Gupta VA, Stone J, Weiss A. A diacylglycerolprotein kinase C-RasGRP1 pathway directs Ras activation upon antigen receptor stimulation of T cells. Mol Cell Biol. 2005;25(11):4426-41.

62. Shahnazari S, Yen WL, Birmingham CL, Shiu J, Namolovan A, Zheng YT, Nakayama K, Klionsky DJ, Brumell JH. A diacylglycerol-dependent signaling pathway contributes to regulation of antibacterial autophagy. Cell Host Microbe. 2010;8(2):137-46.

63. Ulloa G, Hamati F, Dick A, Fitzgerald J, Mantell J, Verkade P, Collinson L, Arkill K, Larijani B, Poccia D. Lipid species affect morphology of endoplasmic reticulum: a sea urchin oocyte model of reversible manipulation. J Lipid Res. 2019;60(11):1880-91.

64. Jun Y, Fratti RA, Wickner W. Diacylglycerol and its formation by phospholipase C regulate Rab- and SNARE-dependent yeast vacuole fusion. J Biol Chem. 2004:279(51):53186-95.

65. Miner GE, Starr ML, Hurst LR, Fratti RA. Deleting the DAG kinase Dgk1 augments yeast vacuole fusion through increased Ypt7 activity and altered membrane fluidity. Traffic. 2017;18(5):315-29.

66. Rockenfeller P, Smolnig M, DiessI J, Bashir M, Schmiedhofer V, Knittelfelde O, Ring J, Franz J, Foessl I, Khan MJ, et al. Diacylglycerol triggers Rim101 pathway-dependent necrosis in yeast: a model for lipotoxicity. Cell Death Differ. 2018;25(4):767-83.

67. Barbosa AD, Sembongi H, Su WM, Abreu S, Reggiori F, Carman GM Siniossoglou S. Lipid partitioning at the nuclear envelope controls membrane biogenesis. Mol Biol Cell. 2015;26(20):3641-57.

68. Petschnigg J, Wolinski H, Kolb D, Zellnig G, Kurat CF, Natter K, Kohlwein SD. Good fat, essential cellular requirements for triacylglycerol synthesis to maintain membrane homeostasis in yeast. J Biol Chem. 2009;284(45): 30981-93.

69. Chitraju C, Walther TC, Farese RV Jr. The triglyceride synthesis enzymes DGAT1 and DGAT2 have distinct and overlapping functions in adipocytes. J Lipid Res. 2019:60(6):1112-20.

70. Chitraju C, Mejhert N, Haas JT, Diaz-Ramirez LG, Grueter CA, Imbriglio JE, Pinto S, Koliwad SK, Walther TC, Farese RV Jr. Triglyceride synthesis by DGAT1 protects adipocytes from lipid-induced ER stress during lipolysis. Cell Metab. 2017:26(2):407-18 e403.

71. Campomanes $P$, Zoni $V$, Vanni S. Local accumulation of diacylglycerol alters membrane properties nonlinearly due to its transbilayer activity. Commun Chem. 2019;2(72). https://doi.org/10.1038/s42004-019-0175-7.
72. McMahon HT, Boucrot E. Membrane curvature at a glance. J Cell Sci. 2015 128(6):1065-70

73. Riske KA, Dobereiner HG. Diacylglycerol-rich domain formation in giant stearoyl-oleoyl phosphatidylcholine vesicles driven by phospholipase C activity. Biophys J. 2003;85(4):2351-62.

74. Ejsing CS, Sampaio JL, Surendranath V, Duchoslav E, Ekroos K, Klemm RW, Simons K, Shevchenko A. Global analysis of the yeast lipidome by quantitative shotgun mass spectrometry. Proc Natl Acad Sci U S A. 2009; 106(7):2136-41.

75. Li D, Song JZ, Shan MH, Li SP, Liu W, Li H, Zhu J, Wang Y, Lin J, Xie Z. A fluorescent tool set for yeast Atg proteins. Autophagy. 2015:11(6):954-60.

76. Zhu J, Zhang ZT, Tang SW, Zhao BS, Li H, Song JZ, Li D, Xie Z. A validated set of fluorescent-protein-based markers for major organelles in yeast (Saccharomyces cerevisiae). MBio. 2019;10(5):e01691-19. https://doi.org/10. 1128/mBio.01691-19.

77. Gueldener U, Heinisch J, Koehler GJ, Voss D, Hegemann JH. A second set of loxP marker cassettes for Cre-mediated multiple gene knockouts in budding yeast. Nucleic Acids Res. 2002;30(6):e23

78. Longtine MS, McKenzie A 3rd, Demarini DJ, Shah NG, Wach A, Brachat A, Philippsen P, Pringle JR. Additional modules for versatile and economical PCR-based gene deletion and modification in Saccharomyces cerevisiae. Yeast. 1998:14(10):953-61.

79. Huang WP, Shintani T, Xie Z. Assays for autophagy I: the Cvt pathway and nonselective autophagy. Methods Mol Biol. 2014;1163:153-64.

80. Zhu M, Zhou PP, Yu LJ. Extraction of lipids from Mortierella alpina and enrichment of arachidonic acid from the fungal lipids. Bioresour Technol. 2002;84(1):93-5

81. Fuchs B, Popkova Y, Süß R, Schiller J. Separation of (phospho)lipids by thinlayer chromatography. In: Poole CF, editor. Instrumental thin-layer chromatography. Elsevier; 2014. p. 375-405.

82. Nakamura K, Handa S. Coomassie brilliant blue staining of lipids on thinlayer plates. Anal Biochem. 1984;142(2):406-10.

83. Wang M, Hayakawa J, Yang K, Han X. Characterization and quantification of diacylglycerol species in biological extracts after one-step derivatization: a shotgun lipidomics approach. Anal Chem. 2014;86(4):2146-55.

\section{Publisher's Note}

Springer Nature remains neutral with regard to jurisdictional claims in published maps and institutional affiliations.

Ready to submit your research? Choose BMC and benefit from:

- fast, convenient online submission

- thorough peer review by experienced researchers in your field

- rapid publication on acceptance

- support for research data, including large and complex data types

- gold Open Access which fosters wider collaboration and increased citations

- maximum visibility for your research: over $100 \mathrm{M}$ website views per year

At BMC, research is always in progress.

Learn more biomedcentral.com/submissions 